\title{
Hyperaccretion Disks around Neutron Stars
}

\author{
Dong Zhang and Z. G. Dai \\ Department of Astronomy, Nanjing University, Nanjing 210093, China; dzg@nju.edu.cn \\ (Accepted for publication in The Astrophysical Journal August 20, 2008, v683n2 issue)
}

\begin{abstract}
It is usually proposed that hyperaccretion disks surrounding stellar-mass black holes at an accretion rate of a fraction of one solar mass per second, which are produced during the mergers of double compact stars or the collapses of massive stars, are central engines of gamma-ray bursts (GRBs). In some origin/afterglow models, however, newborn compact objects are invoked to be neutron stars rather than black holes. Thus, hyperaccretion disks around neutron stars seem to exist in some GRBs. Such disks may also occur in type-II supernovae. In this paper we study the structure of a hyperaccretion disk around a neutron star. Because of the effect of a stellar surface, the disk around a neutron star must be different from that of a black hole. Clearly, far from the neutron star, the disk may have a flow similar to the black hole disk, if their accretion rate and central object mass are the same. Near the compact object, the heat energy in the black-hole disk may be advected inward to the event horizon, but the heat energy in the neutron star disk must be eventually released via neutrino emission because the stellar surface prevents any heat energy from being advected inward. Accordingly, an energy balance between heating and cooling would be built in an inner region of the neutron star disk, which could lead to a self-similar structure of this region. We therefore consider a steady-state hyperaccretion disk around a neutron star, and as a reasonable approximation, divide the disk into two regions, which are called inner and outer disks. The outer disk is similar to that of a black hole and the inner disk has a self-similar structure. In order to study physical properties of the entire disk clearly, we first adopt a simple model, in which some microphysical processes in the disk are simplified, following Popham et al. and Narayan et al. Based on these simplifications, we analytically and numerically investigate the size of the inner disk, the efficiency of neutrino cooling, and the radial distributions of the disk density, temperature and pressure. We see that, compared with the black-hole disk, the neutron star disk can cool more efficiently and produce a much higher neutrino luminosity. Finally, we consider an elaborate model with more physical considerations about the thermodynamics and microphysics in the
\end{abstract}


neutron star disk (as recently developed in studying the neutrino-cooled disk of a black hole), and compare this elaborate model with our simple model. We find that most of the results from these two models are basically consistent with each other.

Subject headings: accretion: accretion disks — black holes — gamma rays: bursts — neutrinos — stars: neutron

\section{Introduction}

Gamma-ray bursts (GRBs) are commonly divided into two classes: short-duration, hardspectrum bursts, and long-duration, soft-spectrum bursts. The observations have provided growing evidence that short bursts result from the mergers of compact star binaries and long bursts originate from the collapses of massive stars (for recent reviews see Zhang \& Mészáros 2004; Piran 2004; Mészáros 2006; Nakar 2007). It is usually assumed that both a compact-star merger and a massive-star collapse give rise to a central black hole and a debris torus around it. The torus has a mass of about $0.01-1 M_{\odot}$ and a large angular momentum enough to produce a transient accretion disk with a huge accretion rate up to $\sim 1.0 M_{\odot} \mathrm{s}^{-1}$. The accretion timescale is short, e.g., a fraction of one second after the merger of two neutron stars, and tens of seconds if a disk forms due to fallback of matter during the collapse process.

The hyperaccretion disk around a black hole is extremely hot and dense. The optical depth of the accreting gas is so enormous that radiation is trapped inside the disk and can only be advected inward. However, in some cases, this hot and dense disk can be cooled via neutrino emission (Narayan et al. 1992). According to the disk structure and different cooling mechanisms, flows in the disk fall into three types: advection-dominated accretion flows (ADAFs), convection-dominated accretion flows (CDAFs), and neutrino-dominated accretion flows (NDAFs). The first two types of flow are radiatively inefficient (Narayan et al. 1998, 2000, 2001) and the final type cools the disk efficiently via neutrino emission (Popham et al. 1999; Di Matteo et al. 2002). In view of effects of these three flows, hyperaccretion disks around black holes have been studied both analytically and numerically (e.g., see Popham et al. 1999; Narayan et al. 2001; Kohri \& Mineshige 2002; Di Matteo et al. 2002; Gu et al. 2006; Chen \& Beloborodov 2007; Liu et al. 2007; Janiuk et al. 2007).

However, newborn neutron stars have been invoked to be central engines of GRBs in some origin/afterglow models. First, the discovery of X-ray flares by Swift implies that the central engines of some GRBs are in a long-living activity (at least hundreds of seconds) 
after the bursts (Zhang 2007). This provides a challenge to conventional hyperaccretion disk models of black holes. Recently, Dai et al. (2006) argued that newborn central compact objects in the GRBs could be young neutron stars, at least transiently-existing neutron stars, rather than black holes (for alternative models see Perna et al. 2006 and Proga \& Zhang 2006). These neutron stars may have high angular momentum and their maximum mass may be close to or slightly larger than the upper mass limit of nonrotating TolmanOppenheimer-Volkoff neutron stars. According to this argument, Dai et al. (2006) explained X-ray flares of short GRBs as being due to magnetic reconnection-driven events from highlymagnetized millisecond pulsars. It is thus reasonable to assume a unified scenario: a prompt burst originates from a highly-magnetized, millisecond-period neutron star surrounded by a transient hyperaccretion disk, and subsequent X-ray flares are due to a series of magnetic activities of the neutron star.

Second, the shallow decay phase of X-ray afterglows about several hundreds of seconds after a sizable fraction of GRBs discovered by Swift has been understood as arising from long-lasting energy injection to relativistic forward shocks (Zhang 2007). It was proposed before Swift observations that pulsars in the unified scenario mentioned above can provide energy injection to a forward shock through magnetic dipole radiation, leading to flattening of an afterglow light curve (Dai \& Lu 1998a; Zhang \& Mészáros 2001; Dai 2004). Recent model fitting (Fan \& Xu 2006; Yu \& Dai 2007) and data analysis (Liang et al. 2007) indeed confirm this result. An ultra-relativistic pulsar wind could be dominated by electron/positron pairs and its interaction with a postburst fireball gives rise to a reverse shock and forward shock (Dai 2004). The high-energy emission due to inverse-Compton scattering in these shocks is significant enough to be detectable with the upcoming Gamma-ray Large-Area Space Telescope (Yu et al. 2007).

Third, we note that, in some origin models of GRBs (e.g., Kluźniak \& Ruderman 1998; Dai \& Lu 1998b; Wheeler et al. 2000; Wang et al. 2000; Paczyński \& Haensel 2005), highlymagnetized neutron stars or strange quark stars surrounded by hyperaccretion disks resulting from fallback of matter could occur during the collapses of massive stars or the mergers of two neutron stars. A similar neutron star was recently invoked in numerical simulations of Mazzali et al. (2006) and data analysis of Soderberg et al. (2006) to understand the properties of supernova SN 2006aj associated with GRB 060218. In addition, hyperaccretion disks could also occur in type-II supernovae if fall-back matter has angular momentum. It would be expected that such disks play an important role in supernova explosions via neutrino emission, similar to some effects reviewed by Bethe (1990).

Based on these motivations, we here investigate a hyperaccretion disk around a neutron star. To our knowledge, this paper is the first to study hyperaccretion disks around neutron 
stars related possibly with GRBs. Chevalier (1996) discussed the structure of dense and neutrino-cooled disks around neutron stars. He considered neutrino cooling due to electronpositron pair annihilation, which is actually much less important than the cooling due to electron-positron pair capture in the hyperaccreting case of our interest, since the accretion rate assumed in Chevalier $(1996)\left(\sim M_{\odot} \mathrm{yr}^{-1}\right)$ is much less than that of our concern $(\sim$ $\left.0.1 M_{\odot} \mathrm{s}^{-1}\right)$. In this paper we consider several types of neutrino cooling by using elaborate formulae developed in recent years. In addition, we focus on some differences between black hole and neutron star hyperaccretion. A main difference is that, the internal energy in an accretion flow may be advected inward into the event horizon without any energy release if the central object is a black hole, but the internal energy must be eventually released from the disk if the central object is a neutron star, because the stellar surface prevents any heat energy from being advected inward into the star. Since the accretion rate is always very high, the effective cooling mechanism in the disk is still neutrino emission, and as a result, the efficiency of neutrino cooling of the entire disk around a neutron star should be higher than that of a black hole disk.

There have been a lot of works to study accretion onto neutron stars in binary systems (e.g., Shapiro \& Salpeter 1975; Kluźniak \& Wilson 1991; Medvedev \& Narayan 2001; Frank et al. 2002) and supernova explosions (e.g., Chevalier 1989, 1996; Brown \& Weingartner 1994; Kohri et al. 2005). In the supernova case, spherically symmetric accretion onto neutron stars (the so-called Bondi accretion) was investigated in detail. In particular, Kohri et al. (2005) tried to use the hyperaccretion disk model with an outflow wind to explain supernova explosions. In the binary systems, as the accretion rate is not larger than the Eddington accretion rate $\left(\sim 10^{-8} M_{\odot} \mathrm{yr}^{-1}\right)$, the physical properties of the disk must be very different from those of a hyperaccretion disk discussed here.

This paper is organized as follows: in $\S 2$, we describe a scheme of our study of the structure of a quasi-steady disk. We propose that the disk around a neutron star can be divided into two regions: inner and outer disks. Table 1 gives the notation and definition of some quantities in this paper. In order to give clear physical properties, we first adopt a simple model in $\S 3$, and give both analytical and numerical results of the disk properties. In $\S 4$, we study the disk using a state-of-the-art model with lots of elaborate considerations about the thermodynamics and microphysics, and compare results from this elaborate model with those from the simple model. $§ 5$ presents conclusions and discussions. 


\section{Description of Our Study Scheme}

\subsection{Motivations of a two-region disk}

We study the quasi-steady structure of an accretion disk around a neutron star with a weak outflow. We take an accretion rate to be a parameter. For the accretion rates of interest to us, the disk flow may be an ADAF or NDAF. In this paper, we do not consider a CDAF. Different from the disk around a black hole, the disk around a neutron star should eventually release the gravitational binding energy of accreted matter (which is converted to the internal energy of the disk and the rotational kinetic energy) more efficiently.

The energy equation of the disk is (Frank et al. 2002)

$$
\Sigma v_{r} T \frac{d s}{d r}=Q^{+}-Q^{-}
$$

where $\Sigma$ is the surface density of the disk, $v_{r}$ is the radial velocity, $T$ is the temperature, $s$ is the entropy per unit mass, $r$ is the radius of a certain position in the disk, and $Q^{+}$ and $Q^{-}$are the energy input (heating) and the energy loss (cooling) rate in the disk. From the point of view of evolution, the structure of a hyperaccretion disk around a neutron star should be initially similar to that of the disk around a black hole, because the energy input is mainly due to the local viscous dissipation, i.e., $Q^{+}=Q_{\mathrm{vis}}^{+}$. However, since the stellar surface prevents the matter and heat energy in the disk from advection inward any more, a region near the compact object should be extremely dense and hot as accretion proceeds. Besides the local viscous heating, this inner region can also be heated by the energy $\left(Q_{\text {adv }}^{+}\right)$ advected from the outer region of the disk. Thus, the heat energy in this region may include both the energy generated by itself and the energy advected from the outer region, that is, we can write $Q^{+}=Q_{\mathrm{vis}}^{+}+Q_{\mathrm{adv}}^{+}$. Initially, such a region is so small (i.e., very near the compact star surface) that it cannot be cooled efficiently for a huge accretion rate $\left(\sim 0.01-1 M_{\odot} \mathrm{s}^{-1}\right)$. As a result, it has to expand its size until an energy balance between heating and cooling is built in this inner region. Such an energy balance can be expressed by $Q^{+}=Q^{-}$in the inner region of the disk.

Once this energy balance is built, the disk is in a steady state as long as the accretion rate is not significantly changed. For such a steady disk, therefore, the structure of the outer region is still similar to that of the disk around a black hole, but the inner region has to be hotter and denser than the disk around a black hole, and could have a different structure from both its initial structure and the outer region that is not affected by the neutron star surface.

Based on the above consideration, the hyperaccretion disk around a neutron star could have two different regions. In order to discuss their structure using a mathematical method 
clearly, as a reasonable approximation, we here divide the steady accretion disk into an inner region and an outer region, called inner and outer disks respectively. The outer disk is similar to that of a black hole. The inner disk, depending on its heating and cooling mechanisms discussed above, should satisfy the entropy conservation condition $T d s / d r \propto Q^{+}-Q^{-}=0$, and thus we obtain $P \propto \rho^{\gamma}$, where $P$ and $\rho$ are the pressure and the density of the disk, and $\gamma$ is the adiabatic index of the disk gas. Also, the radial momentum equation is

$$
\left(\Omega^{2}-\Omega_{K}^{2}\right) r-\frac{1}{\rho} \frac{d}{d r}\left(\rho c_{s}^{2}\right)=0
$$

where $\Omega$ and $\Omega_{K}$ are the angular velocity and Keplerian angular velocity of the inner disk, and $c_{s}=\sqrt{P / \rho}$ is the isothermal sound speed. We here neglect the radial velocity term $v_{r} d v_{r} / d r$ since we consider the situation $v_{r} d v_{r} / d r \ll\left|\Omega^{2}-\Omega_{K}^{2}\right| r$, which can still be satisfied when $v_{r} \ll r \Omega_{K}$ with $\Omega \sim \Omega_{K}$ but $\left|\Omega-\Omega_{K}\right| \geq v_{r} / r$. Equation (2) gives $\Omega \propto r^{-3 / 2}$ and $c_{s} \propto r^{-1 / 2}$. Moreover, from the continuity equation, we have $v_{r} \propto(\rho r H)^{-1}$ with the disk's half-thickness $H=c_{s} / \Omega \propto r$. Thus we can derive a self-similar structure in the inner region of the hyperaccretion disk of a neutron star,

$$
\rho \propto r^{-1 /(\gamma-1)}, P \propto r^{-\gamma /(\gamma-1)}, v_{r} \propto r^{(3-2 \gamma) /(\gamma-1)},
$$

This self-similar structure has been given by Chevalier (1989) and Brown \& Weingartner (1994) for Bondi accretion under the adiabatic condition and by Medvedev \& Narayan (2001) and Medvedev (2004) for disk accretion under the entropy conservation condition. In addition, if the gas pressure is dominated in the disk, we have $\gamma=5 / 3$ so that equation (3) becomes $\rho \propto r^{-3 / 2}, P \propto r^{-5 / 2}$, and $v_{r} \propto r^{-1 / 2}$, which have been discussed by Spruit et al. (1987) and Narayan \& Yi (1994).

An important problem we next solve is to determine the size of the inner disk. Since the total luminosity of neutrinos emitted from the whole disk significantly varies with the inner-region size, we can estimate the inner region size by solving an energy balance between neutrino cooling and heating in the entire disk. The details will be discussed in $§ 2.3$.

Finally, we focus on two problems in this subsection. First, we have to discuss a physical condition of the boundary layer between the outer and inner disks. There are two possible boundary conditions. One condition is to assume that a stalled shock exists at the boundary layer. Under this assumption, the inner disk is a post-shock region, and its pressure, temperature and density just behind the shock are much higher than those of the outer disk in front of the shock. The other condition is to assume that no shock exists in the disk, and that all the physical variables of two sides of this boundary change continuously. We now have to discuss which condition is reasonable.

Let us assume the mass density, pressure, radial velocity, and internal energy density of the outer disk along the boundary layer to be $\rho_{1}, P_{1}, v_{1}$, and $u_{1}$, and the corresponding 
physical variables of the inner disk to be $\rho_{2}, P_{2}, v_{2}$, and $u_{2}$ at the same radius. Thus the conservation equations are

$$
\begin{aligned}
& \rho_{1} v_{1}=\rho_{2} v_{2} \\
& P_{1}+\rho_{1} v_{1}^{2}=P_{2}+\rho_{2} v_{2}^{2} \\
& \left(u_{1}+P_{1}+\rho_{1} v_{1}^{2} / 2\right) / \rho_{1}=\left(u_{2}+P_{2}+\rho_{2} v_{2}^{2} / 2\right) / \rho_{2} .
\end{aligned}
$$

From the Rankine-Hugoniot relations, we know that if $P_{2} \gg P_{1}$, the densities of two sides of the boundary layer are discontinuous, which means that a strong shock exists between the inner and outer disks. On the other hand, if $P_{1} \sim P_{2}$, we can obtain $\rho_{1} \sim \rho_{2}$, which means that only a very weak shock forms at this boundary layer, or we can say that no shock exists. Therefore, we compare $P_{1}$ and $\rho_{1} v_{1}^{2}$ of the outer disk. If $P_{1} \ll \rho_{1} v_{1}^{2}$ or $c_{s} \ll v_{1}$, we can assume that a stalled shock exists at the boundary layer, i.e., the first boundary condition is correct. If $P_{1} \gg \rho_{1} v_{1}^{2}$ or $c_{s} \gg v_{1}$, otherwise, we consider $P_{1} \sim P_{2}$, and thus no shock exists. In $\S 3$ and $\S 4$, we will use this method to discuss which boundary condition is reasonable.

Second, what we want to point out is that the effect of the magnetic field of the central neutron star is not considered in this paper. We estimate the order of magnitude of the Alfven radius by using the following expression (Frank et al 2002), $r_{A} \simeq 2.07 \times 10^{4} \dot{m}_{d}^{-2 / 7} m^{-1 / 7} \mu_{30}^{4 / 7}$, where $\dot{m}_{d}=\dot{M} / 0.01 M_{\odot} \mathrm{s}^{-1}$ is the accretion rate, $m=M / 1.4 M_{\odot}$ is the mass of the neutron star, and $\mu_{30}$ is the magnetic moment of the neutron star in units of $10^{30} \mathrm{G} \mathrm{cm}^{3}$. Let $r_{*}$ be the neutron star radius. If the stellar surface magnetic field $B_{s} \leq B_{s, \mathrm{cr}}=$ $2.80 \times 10^{22} \dot{m}_{d}^{1 / 2} m^{1 / 4} r_{*}^{-5 / 4} \mathrm{G}$ or $B_{s} \leq B_{s, \mathrm{cr}}=0.89 \times 10^{15} \dot{m}_{d}^{1 / 2} m^{1 / 4} \mathrm{G}$ when $r_{*}=10^{6} \mathrm{~cm}$, then the Alfven radius $r_{A}$ is smaller than $r_{*}$. The critical value $B_{s, \text { cr }}$ increases with in-

creasing the accretion rate. This implies that the stellar surface magnetic field affects the structure of the disk significantly if $B_{s} \geq B_{s, \mathrm{cr}} \sim 10^{15}-10^{16} \mathrm{G}$ for typical accretion rates. Therefore, we assume a neutron star with surface magnetic field weaker than $B_{s, \mathrm{cr}}$ in this paper. This assumption is consistent with some GRB-origin models such as Kluźniak \& Ruderman (1998), Dai \& Lu (1998b), Wang et al. (2000), Paczyński \& Haensel (2005), and Dai et al. (2006), because these models require a neutron star or strange quark star with surface magnetic field much weaker than $B_{s, \mathrm{cr}}$.

\subsection{Structure of the outer disk}

Here we discuss the structure of the outer disk based on the Newtonian dynamics and the standard $\alpha$-viscosity disk model for simplicity. The structure of the hyperaccretion disk around a stellar-mass black hole has been discussed in many previous works. The method and equations we use here are similar to those in the previous works since the outer disk is very similar to the disk around a black hole. 
We approximately consider the angular velocity of the outer disk to be the Keplerian value $\Omega_{K}=\sqrt{G M / r^{3}}$. The velocity $\Omega_{K}$ should be modified in a relativistic model of accretion disks (Popham et al. 1999; Chen \& Beloborodov 2007), but we do not consider it in this paper. We can write four equations to describe the outer disk, i.e., the continuity equation, the energy equation, the angular momentum equation and the equation of state.

The continuity equation is

$$
\dot{M}=4 \pi r \rho v_{r} H \equiv 2 \pi r \Sigma v_{r},
$$

where the notations of the physical quantities have been introduced in $§ 2.1$.

In the outer disk, the heat energy could be advected inward and we take $Q_{\text {adv }}^{-}=$ $(1 / 2) \Sigma T v_{r} d s / d r$ to be the quantity of the energy advection rate, where the factor $1 / 2$ is added because we only study the vertically-integrated disk over a half-thickness $H$. Thus the energy-conservation equation (11) is rewritten as

$$
Q^{+}=Q_{\mathrm{rad}}^{-}+Q_{\mathrm{adv}}^{-}+Q_{\nu}^{-}
$$

The quantity $Q^{+}$in equation (6) is the viscous heat energy generation rate per unit surface area. According to the standard viscosity disk model, we have

$$
Q^{+}=\frac{3 G M \dot{M}}{8 \pi r^{3}} f
$$

where $f=1-\left(r_{*} / r\right)^{1 / 2}$ (Frank et al. 2002).

The quantity $Q_{\text {rad }}^{-}$in equation (6) is the photon cooling rate per unit surface area of the disk. Since the disk is extremely dense and hot, the optical depth of photons is always very large and thus we can take $Q_{\text {rad }}^{-}=0$ as a good approximation.

The entropy per unit mass of the disk is (similar to Kohri \& Mineshige 2002)

$$
s=s_{\text {gas }}+s_{\text {rad }}=\frac{S_{\text {gas }}}{\rho}+\frac{S_{\text {rad }}}{\rho}=\sum_{i} n_{i}\left\{\frac{5}{2} \frac{k_{B}}{\rho}+\frac{k_{B}}{\rho} \ln \left[\frac{\left(2 \pi k_{B} T\right)^{3 / 2}}{h^{3} n_{i}}\right]\right\}+S_{0}+\frac{2}{3} g_{*} \frac{a T^{3}}{\rho},
$$

where the summation runs over nucleons and electrons, $k_{B}$ is the Boltzmann constant, $h$ is the Planck constant, $a$ is the radiation constant, $S_{0}$ is the integration constant of the gas entropy, and the term $2 g_{*} a T^{3} / 3 \rho$ is the entropy density of the radiation with $g_{*}=2$ for photons and $g_{*}=11 / 2$ for a plasma of photons and relativistic $e^{+} e^{-}$pairs. We assume that electrons and nucleons have the same temperature. Then we use equation (8) to calculate $d s / d r$ and approximately take $d T / d r \approx T / r$ and $d \rho / d r \approx \rho / r$ to obtain the energy advection rate,

$$
Q_{\mathrm{adv}}^{-}=v_{r} T \frac{\Sigma}{2 r}\left[\frac{R}{2}\left(1+Y_{e}\right)+\frac{4}{3} g_{*} \frac{a T^{3}}{\rho}\right]
$$


where the gas constant $R=8.315 \times 10^{7} \mathrm{ergs} \mathrm{mole}^{-1} \mathrm{~K}^{-1}$ and $Y_{e}$ is the ratio of electron to nucleon number density. The first term in the right-hand bracket of equation (9) comes from the contribution of the gas entropy and the second term from the contribution of radiation.

The quantity $Q_{\nu}^{-}$in equation (6) is the neutrino cooling rate per unit area. The expression of $Q_{\nu}^{-}$will be discussed in detail in $\S 3$ and $\S 4$.

In this paper we ignore the cooling term of photodisintegration $Q_{\text {photodis }}^{-}$, and approximately take the free nucleon fraction $X_{\text {nuc }} \approx 1$. For the disks formed by the collapses of massive stars, the photodisintegration process that breaks down $\alpha$-particles into neutrons and protons is important in a disk region at very large radius $r$. However, the effect of photodisintegration becomes less significant for a region at small radius, where contains less $\alpha$-particles 1 . On the other hand, for the disks formed by the mergers of double compact stars, there will be rare $\alpha$-particles in the entire disk. As a result, we reasonably take all the nucleons to be free $\left(X_{\text {nuc }} \approx 1\right)$ and neglect the photodisintegration process, since we mainly focus on small disks or small regions of the disks (as we will mention in $\S 3.4$ with the outer boundary $r_{\text {out }}$ to be $150 \mathrm{~km}$.)

Furthermore, the angular momentum conservation and the equation of sate can be written as

$$
\begin{gathered}
\nu \Sigma=\frac{\dot{M}}{3 \pi} f, \\
P=P_{e}+P_{\text {nuc }}+P_{\text {rad }}+P_{\nu},
\end{gathered}
$$

where $\nu=\alpha c_{s} H$ in equation (10) is the kinematic viscosity and $\alpha$ is the classical viscosity parameter, and $P_{e}, P_{\text {nuc }}, P_{\text {rad }}$ and $P_{\nu}$ in equation (11) are the pressures of electrons, nucleons, radiation and neutrinos. In $\S 3$ we will consider the pressure of electrons in extreme cases and in $\S 4$ we will calculate the $e^{ \pm}$pressure using the exact Fermi-Dirac distribution function and the condition of $\beta$-equilibrium.

Equations (5), (6),$(10)$ and (11) are the basic equations to solve the structure of the outer disk, which is important for us to study the inner disk.

\footnotetext{
${ }^{1}$ Kohri et al. (2005), Chen \& Beloborodov (2007), and Liu et al. (2007) discussed the value of nucleon fraction $X_{\text {nuc }}$ and the effect of photodisintegration as a function of radius for particular parameters such as the accretion rate and the viscosity parameter $\alpha$. The former two papers show that $X_{\text {nuc }} \approx 1$ and $Q_{\text {photodis }} \approx 0$ for $r \leq 10^{2} r_{g}$. Although the value of $X_{\text {nuc }}$ from Liu et al. (2007) is somewhat different from the previous works, the ratio of $Q_{\text {photodis }}^{-} / Q^{+}$in their work also drops dramatically for $r \leq 10^{2} r_{g}$. Therefore, it is convenient for us to neglect the photodisintegration process for $r \leq 10^{2} r_{g}$ or $r \leq 400 \mathrm{~km}$ for the central star mass $M=1.4 M_{\odot}$.
} 


\subsection{Self-similar structure of the inner disk}

In $§ 2.1$ we introduced a self-similar structure of the inner disk, and described the method to determine the size of the inner disk. Now we will establish the energy conservation equation in the inner disk. We assume that $\tilde{r}$ is the radius of the boundary layer between the inner and outer disks, and that $\tilde{\rho}, \tilde{P}$, and $\tilde{v}_{r}$ are the density, pressure and radial velocity of the inner disk just at the boundary layer respectively. From equation (3), thus, the variables in the inner disk at any given radius $r$ can be written by

$$
\rho=\tilde{\rho}(\tilde{r} / r)^{1 /(\gamma-1)}, P=\tilde{P}(\tilde{r} / r)^{\gamma /(\gamma-1)}, v_{r}=\tilde{v}_{r}(\tilde{r} / r)^{(2 \gamma-3) /(\gamma-1)} .
$$

We take the outer radius of the accretion disk to be $r_{\text {out }}$. The total energy per unit time that the outer disk advects into the inner disk is (Frank et al. 2002)

$$
\dot{E}_{\mathrm{adv}}=\left(1-\bar{f}_{\nu}\right) \frac{3 G M \dot{M}}{4}\left\{\frac{1}{\tilde{r}}\left[1-\frac{2}{3}\left(\frac{r_{*}}{\tilde{r}}\right)^{1 / 2}\right]-\frac{1}{r_{\text {out }}}\left[1-\frac{2}{3}\left(\frac{r_{*}}{r_{\text {out }}}\right)^{1 / 2}\right]\right\},
$$

where $\bar{f}_{\nu}$ is the average neutrino cooling efficiency of the outer disk,

$$
\bar{f}_{\nu}=\frac{\int_{\tilde{r}}^{r_{\text {out }}} Q_{\nu}^{-} 2 \pi r d r}{\int_{\tilde{r}}^{r_{\text {out }}} Q^{+} 2 \pi r d r}
$$

If the outer disk flow is mainly an ADAF, $Q_{\nu}^{-} \ll Q^{+}$, then $\bar{f}_{\nu} \sim 0$; if the outer disk flow is mainly an NDAF, $Q_{\nu}^{-} \gg Q_{\text {rad }}^{-}$and $Q_{\nu}^{-} \gg Q_{\text {adv }}^{-}$, we have $\bar{f}_{\nu} \sim 1$. The heat energy in the inner disk should be released more efficiently than the outer disk, we can still approximately take $\Omega \simeq \Omega_{K}$ in the inner disk, and the maximum power that the inner disk can release is

$$
\begin{aligned}
L_{\nu, \text { max }} \approx & \frac{3 G M \dot{M}}{4}\left\{\frac{1}{3 r_{*}}-\frac{1}{r_{\text {out }}}\left[1-\frac{2}{3}\left(\frac{r_{*}}{r_{\text {out }}}\right)^{1 / 2}\right]\right\} \\
& -\bar{f}_{\nu} \frac{3 G M \dot{M}}{4}\left\{\frac{1}{\tilde{r}}\left[1-\frac{2}{3}\left(\frac{r_{*}}{\tilde{r}}\right)^{1 / 2}\right]-\frac{1}{r_{\text {out }}}\left[1-\frac{2}{3}\left(\frac{r_{*}}{r_{\text {out }}}\right)^{1 / 2}\right]\right\},
\end{aligned}
$$

where we have integrated vertically over the half-thickness. The first term in the right-hand of this equation is the total heat energy per unit time of the entire disk, and the second term is the power taken away through neutrino cooling in the outer disk. Considering $r_{\text {out }} \gg r_{*}$, we have

$$
L_{\nu, \max } \approx \frac{3 G M \dot{M}}{4}\left\{\frac{1}{3 r_{*}}-\frac{\bar{f}_{\nu}}{\tilde{r}}\left[1-\frac{2}{3}\left(\frac{r_{*}}{\tilde{r}}\right)^{1 / 2}\right]\right\} .
$$

The maximum energy release rate of the inner disk is $G M \dot{M} / 4 r_{*}$ if the outer disk flow is mainly an ADAF and $\bar{f}_{\nu} \sim 0$. This value is just one half of the gravitational binding energy 
and satisfies the Virial theorem. If the outer disk flow is an NDAF, then the energy release of the inner disk mainly results from the heat energy generated by its own.

Following the above consideration, most of the energy generated in the disk around a neutron star is still released from the disk, so we have an energy-conservation equation,

$$
\int_{r_{*}}^{\tilde{r}} Q_{\nu}^{-} 2 \pi r d r=\varepsilon \frac{3 G M \dot{M}}{4}\left\{\frac{1}{3 r_{*}}-\frac{\bar{f}_{\nu}}{\tilde{r}}\left[1-\frac{2}{3}\left(\frac{r_{*}}{\tilde{r}}\right)^{1 / 2}\right]\right\},
$$

where $\varepsilon$ is a parameter that measures the efficiency of the energy release. If the central compact object is a black hole, we have $\varepsilon \approx 0$ and the inner disk cannot exist. If the central compact object is a neutron star, we can take $\varepsilon \approx 1$ and thus we are able to use equation (17) to determine the size of the inner disk.

\section{A Simple Model of the Disk}

In $\S 2$, we gave the equations of describing the structure of a hyperaccretion disk. However, additional equations about microphysics in the disk are needed. In order to see the physical properties of the entire disk clearly, we first adopt a simple model for an analytical purpose. Comparing with $\S 4$, we here adopt a relatively simple treatment with the disk microphysics similar to Popham et al. (1999) and Narayan et al. (2001), and discuss some important physical properties, and then we compare analytical results with numerical ones which are also based on the simple model.

If the disk is optically thin to its own neutrino emission, the neutrino cooling rate can be written as a summation of four terms including the electron-positron pair capture rate, the electron-positron pair annihilation rate, the nucleon bremsstrahlung rate and the plasmon decay rate, that is, $Q_{\nu}^{-}=\left(\dot{q}_{\mathrm{eN}}+\dot{q}_{e^{+} e^{-}}+\dot{q}_{\text {brems }}+\dot{q}_{\text {plasmon }}\right) H$ (Kohri \& Mineshige 2002). We take two major contributions of these four terms and use the approximative formulae: $\dot{q}_{e^{+} e^{-}}=5 \times 10^{33} T_{11}^{9} \mathrm{ergs} \mathrm{cm}^{-3} \mathrm{~s}^{-1}$, and $\dot{q}_{\mathrm{eN}}=9 \times 10^{23} \rho T_{11}^{6} \mathrm{ergs} \mathrm{cm}^{-3} \mathrm{~s}^{-1}$. Thus equation (6) can be rewritten as

$$
\frac{3 G M \dot{M}}{8 \pi r^{3}} f=\frac{\dot{M} T}{4 \pi r^{2}}\left[\frac{R}{2}\left(1+Y_{e}\right)+\frac{22}{3} \frac{a T^{3}}{\rho}\right]+\left(5 \times 10^{33} T_{11}^{9}+9 \times 10^{23} \rho T_{11}^{6}\right) \frac{c_{s}}{\Omega_{K}} .
$$

If neutrinos are trapped in the disk, we use the blackbody limit for the neutrino emission luminosity: $Q_{\nu}^{-} \sim\left(\frac{7}{8} \sigma_{B} T^{4}\right) / \tau$, where $\tau$ is the neutrino optical depth. We approximately estimate the neutrino optical depth as $\left(\dot{q}_{e^{+} e^{-}}+\dot{q}_{\mathrm{eN}}\right) H /\left(4 \times \frac{7}{8} \sigma_{B} T^{4}\right)$.

Moreover, we take the total pressure in the disk to be a summation of three terms $P_{e}$, $P_{\text {nuc }}$ and $P_{\text {rad }}$, and neglect the pressure of neutrinos: $P_{e}=n_{e} k T+K_{1}\left(\rho Y_{e}\right)^{4 / 3}, P_{\text {nuc }}=n_{\text {nuc }} k T$, 
and $P_{\text {rad }}=11 a T^{4} / 12$, where $K_{1}\left(\rho Y_{e}\right)^{4 / 3}$ is the relativistic degeneracy pressure of electrons, $n_{e}$ and $n_{\text {nuc }}$ are the number densities of electrons and nucleons respectively. Here we also neglect the non-relativistic degeneracy pressure of nucleons. We thus obtain

$$
P=P_{e}+P_{\mathrm{nuc}}+P_{\mathrm{rad}}=\rho\left(1+Y_{e}\right) R T+K_{1}\left(\rho Y_{e}\right)^{4 / 3}+\frac{11}{12} a T^{4},
$$

where $K_{1}=\frac{2 \pi h c}{3}\left(\frac{3}{8 \pi m_{p}}\right)^{4 / 3}=1.24 \times 10^{15} \mathrm{cgs}$.

Equations (10), (18) and (19) can be solved for three unknowns (density, temperature and pressure) of the steady outer disk as functions of radius $r$ for four given parameters $\alpha$, $Y_{e}, \dot{M}$ and $M$ in the simple model. Once the density, temperature and pressure profiles are determined, we can present the structure of the outer disk and further establish the size and the structure of the inner disk.

\subsection{The outer disk}

We analytically solve equations (10), (18) and (19) in this subsection. Our method is similar to that of Narayan et al. (2001). However, what is different from their work is that we use the same equations to obtain both ADAF and NDAF solutions in different conditions. Besides, we find that the factor $f=1-\left(r_{*} / r\right)^{1 / 2}$ cannot be omitted because it plays an important role in determining the disk structure. In this subsection, for convenience, we expand the solution range to the entire disk (i.e., $r_{*}<r<r_{\text {out }}$ ) rather than just consider it in the outer region. The size of the inner disk, which depends on the structure of the outer disk, will be solved in $\S 3.2$.

First we show a general picture. If the accretion rate is not very high, most of the energy generated in the disk is advected inward and we call the disk as an advection-dominated disk. As the accretion rate increases, the density and temperature of the disk also increase and the neutrino cooling in some region of the disk becomes the dominant cooling mechanism. Thus we say that this region becomes neutrino-dominated. When the accretion rate is sufficiently large, the disk may totally become neutrino-dominated. Besides the accretion rate, there are some other factors such as the mass of the central neutron star, $M$, and the electron-nucleon ratio, $Y_{e}$, are able to influence the disk structure.

We take the mass of neutron star $M=1.4 m M_{\odot}$, the accretion rate $\dot{M}=\dot{m}_{d} \times$ $0.01 M_{\odot} \mathrm{s}^{-1}, \alpha=0.1 \alpha_{-1}, r=10^{6} r_{6} \mathrm{~cm}, \rho=10^{11} \rho_{11} \mathrm{~g} \mathrm{~cm}^{-3}, T=10^{11} T_{11} \mathrm{~K}$, and $P=$ $10^{29} P_{29} \mathrm{ergs}^{-3}$. In the case that the disk flow is an ADAF with the radiation pressure to 
be dominated, we find that the density and temperature in the disk are

$$
\begin{aligned}
& \rho_{11}=0.0953 \dot{m}_{d} m^{-1 / 2} f^{-1 / 2} \alpha_{-1}^{-1} r_{6}^{-3 / 2} \\
& T_{11}=0.832 m^{1 / 8} \dot{m}_{d}^{1 / 4} f^{1 / 8} \alpha_{-1}^{-1 / 4} r_{6}^{-5 / 8}
\end{aligned}
$$

Also, the pressure of the disk from equation (19) becomes

$$
P_{29}=3.32 m^{1 / 2} \dot{m}_{d} f^{1 / 2} \alpha_{-1}^{-1} r_{6}^{-5 / 2} .
$$

We have to check the validity of the assumption made in deriving the above solution, i.e., we need the relations $Q_{\text {adv }}^{-}>Q_{\nu}^{-}$and $P_{\text {rad }}>P_{\text {gas }}, P_{\text {rad }}>P_{\text {deg }}$ to be satisfied, where $P_{\text {rad }}$ and $P_{\text {deg }}$ are the gas and degeneracy pressure in the disk. Then we get the relations that

$$
\begin{gathered}
r_{6} f^{1 / 5}>2.28 m^{-3 / 5} \dot{m}_{d}^{6 / 5} \alpha_{-1}^{-2} . \\
r_{6} f^{-7 / 3}<74.6\left(1+Y_{e}\right)^{-8 / 3} m^{7 / 3} \dot{m}_{d}^{-2 / 3} \alpha_{-1}^{2 / 3}, \\
r_{6} f^{-7 / 3}<174 m^{7 / 3} \dot{m}_{d}^{-2 / 3} \alpha_{-1}^{2 / 3} Y_{e}^{-8 / 3} .
\end{gathered}
$$

In particular, for a fixed radius $r$, we can rewrite inequation (22) as

$$
\dot{m}_{d}<0.504 m^{1 / 2} \alpha_{-1}^{5 / 3} r_{6}^{5 / 6} f^{1 / 6}
$$

which means that a larger radius allows a larger upper limit of the accretion rate for the radiation-pressure-dominated region. On the other hand, if the parameters $m, \alpha$ and $\dot{m}_{d}$ in some region of the disk do not satisfy inequations (22) or (25), the other types of pressure can exceed the radiation pressure but the region can still be advection-dominated. For an analytical purpose, we want to discuss the range of different types of pressure in two extreme cases where $Y_{e} \sim 1$ or $Y_{e} \ll 1$. From inequation (23), we can see that, since the minimum value of $r_{6} f^{-7 / 3}$ is 19.9 , when $\dot{m}_{d}>0.453 m^{7 / 2} \alpha_{-1}$ for $Y_{e} \sim 1$, or $\dot{m}_{d}>7.25 m^{7 / 2} \alpha_{-1}$ for $Y_{e} \ll 1$, the gas pressure takes over the radiation pressure in the disk and the entire disk becomes gas-pressure-dominated. On the other hand, the degeneracy pressure is larger than the radiation pressure at a very large radius if the electron fraction $Y_{e}$ is not very small. However, we do not consider this situation for an ADAF region of the disk, because the degeneracy pressure, even if larger than the radiation pressure, cannot exceed the gas pressure.

When the gas pressure is dominated and the outer disk flow is still an ADAF, we obtain the density, temperature and pressure of the disk as

$$
\begin{aligned}
& \rho_{11}=0.556\left(1+Y_{e}\right)^{-12 / 11} m^{5 / 11} \dot{m}_{d}^{8 / 11} f^{5 / 11} \alpha_{-1}^{-8 / 11} r_{6}^{-21 / 11} \\
& T_{11}=1.29\left(1+Y_{e}\right)^{-3 / 11} m^{4 / 11} \dot{m}_{d}^{2 / 11} f^{4 / 11} \alpha_{-1}^{-2 / 11} r_{6}^{-8 / 11} \\
& P_{29}=5.98\left(1+Y_{e}\right)^{-4 / 11} m^{9 / 11} \dot{m}_{d}^{10 / 11} f^{9 / 11} \alpha_{-1}^{-10 / 11} r_{6}^{-29 / 11}
\end{aligned}
$$


Similarly, we discuss the validity of the solution (26). The assumption of a gas-pressuredominated ADAF disk $\left(Q_{\mathrm{adv}}^{-}>Q_{\nu}^{-}\right)$requires

$$
r_{6}^{47 / 22} f^{-20 / 11}>128\left(1+Y_{e}\right)^{-26 / 11} m^{29 / 22} \dot{m}_{d}^{10 / 11} \alpha_{-1}^{-21 / 11}
$$

$P_{\text {gas }}>P_{\text {rad }}$ can be written by

$$
r_{6} f^{-7 / 3}>74.6\left(1+Y_{e}\right)^{-8 / 3} m^{7 / 3} \dot{m}_{d}^{-2 / 3} \alpha_{-1}^{2 / 3}
$$

$P_{\text {gas }}>P_{\text {deg }}$ leads to

$$
r_{6} f^{-7 / 3}<8.07 \times 10^{3}\left(1+Y_{e}\right)^{12} Y_{e}^{-44 / 3} m^{7 / 3} \dot{m}_{d}^{-2 / 3} \alpha_{-1}^{2 / 3}
$$

which is satisfied for a large parameter space.

If $Y_{e} \sim 1$ and the parameters $m$ and $\alpha$ make $0.453 m^{7 / 2} \alpha_{-1}<\dot{m}_{d}<1.73 m^{-29 / 20} \alpha_{-1}^{21 / 10}$ valid, the entire disk becomes an advection-dominated disk with the gas pressure to be dominated. However, if $Y_{e} \ll 1$, such a disk cannot exist, since inequation (27) cannot be always satisfied in the entire disk and some region of the disk would become neutrinodominated. Also, when the mass accretion rate $\dot{m}_{d}$ becomes higher, most region of the disk becomes neutrino-dominated.

Furthermore, in the region where neutrino cooling is efficient and the gas pressure dominates over the degeneracy pressure, we have another particular solution

$$
\begin{aligned}
& \rho_{11}=2.38\left(1+Y_{e}\right)^{-9 / 5} m^{17 / 20} \dot{m}_{d} f \alpha_{-1}^{-13 / 10} r_{6}^{-51 / 20} \\
& T_{11}=0.490\left(1+Y_{e}\right)^{1 / 5} m^{1 / 10} \alpha_{-1}^{1 / 5} r_{6}^{-3 / 10} \\
& P_{29}=9.71\left(1+Y_{e}\right)^{-3 / 5} m^{19 / 20} \dot{m}_{d} f \alpha_{-1}^{-11 / 10} r_{6}^{-57 / 20}
\end{aligned}
$$

In this case, the temperature is independent of the accretion rate $\dot{m}_{d}$. Finally we check the gas pressure-dominated assumption. Using equation (30) and assuming $P_{\text {gas }}>P_{\text {deg }}$, we obtain

$$
r_{6} f^{-20 / 33}>3.18 Y_{e}^{80 / 33}\left(1+Y_{e}\right)^{-36 / 11} m^{1 / 3} \dot{m}_{d}^{20 / 33} \alpha_{-1}^{-38 / 33} .
$$

Inequation (31) is always satisfied if $Y_{e} \ll 1$, and thus we can say that the gas pressuredominated assumption is valid. However, if $Y_{e} \sim 1$, a part of the disk becomes degeneracy pressure-dominated if $\dot{m}_{d}>64.5 \alpha_{-1}^{19 / 10} \mathrm{~m}^{-11 / 20}$. In particular, in the case of $Y_{e} \sim 1$ and large accretion rate $\dot{m}_{d}$, a set of solutions on the part of the disk are

$$
\begin{aligned}
& \rho_{11}=1.26 Y_{e}^{-4 / 3} m^{2 / 3} \dot{m}_{d}^{2 / 3} f^{2 / 3} \alpha_{-1}^{-2 / 3} r_{6}^{-2} \\
& T_{11}=0.526 Y_{e}^{4 / 27} m^{13 / 108} \dot{m}_{d}^{1 / 27} f^{1 / 27} \alpha_{-1}^{7 / 54} r_{6}^{-13 / 36} \\
& P_{29}=7.85 Y_{e}^{-4 / 9} m^{8 / 9} \dot{m}_{d}^{8 / 9} f^{8 / 9} \alpha_{-1}^{-8 / 9} r_{6}^{-8 / 3}
\end{aligned}
$$


which describe an NDAF with the degeneracy pressure to be dominated. However, we should remember that in deriving the above solutions we have not considered the constraint of $r>\tilde{r}$.

Here we make a summary of $\S 3.1$. We used an analytical method to solve the density, pressure and temperature of the outer disk based on a simple model discussed at the beginning of $\S 3$. The accretion flow may be ADAF or NDAF with the radiation, gas or degeneracy pressure to be dominated. We fix radius $r$ and show several possible cases in the disk with different accretion rate $\dot{m}_{d}$ in Table 2. Moreover, for different electron fraction $Y_{e}$ and fixing $m=1$ and $\alpha_{-1}=1$, we calculate the upper limit of $\dot{m}_{d}$. If $Y_{e} \sim 1$, the advection-dominated region in the disk can be radiation or gas pressure-dominated, and the neutrino-cooled region can be gas or degeneracy pressure-dominated. However, if $Y_{e} \ll 1$, the gas pressure-dominated region in the ADAF case is very small, and the degeneracy pressure-dominated region cannot exist. In $\S 3.4$ we will obtain similar results by using a numerical method.

The solutions given in this subsection can also be used to discuss the properties of the disk around a black hole. Our analytical solutions of the outer disk are similar to those of Narayan et al. (2001) and Di Matteo et al. (2002), who took $Y_{e}$ to be a parameter. Narayan et al. (2001) found that advection-dominated disks can be radiation or gas pressure-dominated, and neutrino-dominated disks can be gas or degeneracy pressure-dominated instead. This is consistent with our conclusion for $Y_{e} \sim 1$. However, these authors did not consider the factor $f=1-\sqrt{r_{*} / r}$, which is an important factor because a small $r$, as we have mentioned above, can dramatically change the parameter space of the outer disk. Di Matteo et al. (2002) discussed different pressure components (their Fig. 2), which is also consistent with our conclusion. Chen \& Beloborodov (2007) calculated the value of $Y_{e}$ and showed that $Y_{e} \ll 1$ when $r$ is small. According to the above discussion, therefore, the degeneracypressure-dominated region in the NDAF disk cannot exist. This is consistent with Chen \& Beloborodov (2007) that the pressure in a neutrino-cooled disk is dominated by baryons (gas).

However, our analytical results are partly different with Kohri et al. (2005) and Chen \& Beloborodov (2007) which showed that the electron pressure is dominant in some advectiondominated regions of the disk. This difference is mainly because that we take $P_{\text {rad }}=$ $11 a T^{4} / 12$ in our analytical model, which includes the contribution of relativistic electronpositron pairs. However, Kohri et al. (2005) and Chen \& Beloborodov (2007) took $P_{\text {rad }}=$ $a T^{4} / 3$ and calculated the pressure of $e^{+} e^{-}$pairs in the term of electron pressure $P_{e}$. As a result, the radiation pressure we consider in this subsection is actually the pressure of a photon and $e^{+} e^{-}$-pair plasma.

In the following subsection, we will establish the structure of the inner disk depending 
on the outer disk solutions here, and use the value of $\tilde{r}$ to further constrain the solutions that we have obtained.

\subsection{The inner disk}

\subsubsection{Boundary layer between the inner disk and outer disk}

We use the method discussed in $§ 2.1$ and compare the radial velocity with the local speed of sound of the outer disk using the results given in section $§ 3.1$.

The radial velocity of the outer disk at radius $r$ is

$$
v_{1}=\frac{\dot{M}}{2 \pi r \Sigma}=\frac{\dot{M}}{4 \pi r \rho_{1} H}=\frac{\dot{M} \sqrt{G M}}{4 \pi r^{5 / 2} \sqrt{P_{1} \rho_{1}}} .
$$

Hence, we have

$$
\frac{v_{1}^{2}}{c_{s}^{2}} \sim \frac{\rho_{1} v_{1}^{2}}{P_{1}}=\frac{\dot{M}^{2} G M}{16 \pi^{2} r^{5} P_{1}^{2}}=0.0465 \frac{\dot{m}_{d}^{2} m}{r_{6}^{5} P_{1,29}^{2}} .
$$

In the case where the outer disk is $\mathrm{ADAF}$ and the radiation pressure is dominated, using solutions (20) and (21), we have

$$
\frac{v_{1}^{2}}{c_{s}^{2}} \sim 4.22 \times 10^{-3} \alpha_{-1}^{2} f^{-1}
$$

Therefore, we see $v_{1} \ll c_{s}$ for typical values of the parameters.

In the case of ADAF with the gas pressure to be dominated, using solution (26), we have

$$
\frac{v_{1}^{2}}{c_{s}^{2}} \sim 1.302 \times 10^{-3}\left(1+Y_{e}\right)^{8 / 11} m^{-7 / 11} \dot{m}_{d}^{2 / 11} \alpha_{-1}^{20 / 11} f^{-18 / 11} r_{6}^{3 / 11}
$$

For NDAF, using expression (30) to compare the radial velocity with the speed of sound, we have

$$
\frac{v_{1}^{2}}{c_{s}^{2}} \sim 4.94 \times 10^{-4}\left(1+Y_{e}\right)^{6 / 5} m^{-9 / 10} \alpha_{-1}^{11 / 5} f^{-2} r_{6}^{7 / 10}
$$

Note that this ratio is independent of the accretion rate. From equations (36) and (37), we still find that $v_{1} \ll c_{s}$ is always satisfied except for a region very near the stellar surface, where the factor $f$ is very small. This region, however, is so small that it belongs to the inner disk where we have to use the self-similar structure, which we will discuss later in details.

Therefore, for the hyperaccretion disk discussed in this paper, as the disk is extremely hot and dense, the radial velocity is always subsonic. So there is no stalled shock between 
the inner and outer disks. Thus, all physical variables between two sides of the boundary layer between two regions of the disk change continuously. Besides, the rotational velocity is assumed to be the Keplerian value and has no jump at the boundary layer.

\subsubsection{Solution of the inner disk}

We now study the inner disk analytically based on the results given in $§ 3.1$. The main problem that we should solve in this subsection is to determine the size of the inner disk for a range of parameters and to describe the structure of the inner disk. In the case where the radiation pressure is dominated, by using the self-similar structure (12), we obtain the temperature of the inner disk,

$$
T_{11}=\left(\frac{P_{29}}{6.931}\right)^{1 / 4}=1.01 m^{1 / 6} \tilde{\rho}_{11}^{1 / 2} \dot{m}_{d}^{1 / 6} \tilde{f}^{1 / 6} \alpha_{-1}^{-1 / 6} \tilde{r}_{6}^{\frac{2-\gamma}{4(\gamma-1)}} r_{6}^{\frac{-\gamma}{4(\gamma-1)}} .
$$

where $\tilde{r}$ and $\tilde{\rho}$ are the radius and density of the boundary layer between the inner and outer disk, and $\tilde{f}=1-\left(r_{*} / \tilde{r}\right)^{1 / 2}$.

Using the self-similar condition and the above expression of $T_{11}$, we find the total neutrino cooling rate,

$$
\int_{r_{*}}^{\tilde{r}} Q_{\nu}^{-} 2 \pi r d r=10^{51} \times \int 120 \tilde{\rho}_{11}^{7 / 6} m^{5 / 6} \dot{m}_{d}^{4 / 3} \alpha_{-1}^{-4 / 3} \tilde{f}^{4 / 3} \tilde{r}_{6}^{\frac{9-4 \gamma}{2(\gamma-1)}} r_{6}^{\frac{\gamma-6}{2(\gamma-1)}} d r_{6} .
$$

The outer disk flow is mainly an ADAF, using the solution of a radiation pressuredominated ADAF (i.e., solutions 20 and 21), we have the total neutrino cooling rate,

$$
L_{\nu}=1.55 \times 10^{52} \operatorname{ergs~s}^{-1}\left(\frac{\gamma-1}{8-3 \gamma}\right) m^{1 / 4} \dot{m}_{d}^{5 / 2} \tilde{f}^{3 / 4} \alpha_{-1}^{-5 / 2} \tilde{r}_{6}^{\frac{25-15 \gamma}{4(\gamma-1)}}\left(r_{*, 6}^{\frac{3 \gamma-8}{2(\gamma-1)}}-\tilde{r}_{6}^{\frac{3 \gamma-8}{2(\gamma-1)}}\right)
$$

From the energy-conservation equation (17), the position of the boundary layer satisfies the following equation,

$$
\tilde{r}_{6}^{\frac{5(5-3 \gamma)}{4(\gamma-1)}}\left(1-\sqrt{\frac{r_{*, 6}}{\tilde{r}_{6}}}\right)^{3 / 4}\left(r_{*, 6}^{\frac{3 \gamma-8}{2(\gamma-1)}}-\tilde{r}_{6}^{\frac{3 \gamma-8}{2(\gamma-1)}}\right)=0.0597\left(\frac{8-3 \gamma}{\gamma-1}\right) m^{3 / 4} \dot{m}_{d}^{-3 / 2} \alpha_{-1}^{5 / 2} r_{*, 6}^{-1},
$$

where we take $\varepsilon \sim 1$ and $\bar{f}_{\nu} \sim 0$ in equation (17). The left-hand term of equation (41) increases with increasing $\tilde{r}_{6}$, so $\tilde{r}_{6}$ decreases if $\dot{m}_{d}$ increases in the right-hand term of this equation. In other words, the size of the inner disk decreases as the accretion rate increases. From equation (41), we can also see that its solution, $\tilde{r}_{6}$, is independent of $Y_{e}$, and increases with the mass of the central star. In addition, since the gas pressure has its own contribution 
to the disk, the actual adiabatic index $\gamma$ is larger than $4 / 3$, which can makes the solution of equation (41) larger. For an analytical purpose, we assume several different sets of parameters to solve equation (41). Table 3 clearly shows that, in the radiation-pressure-dominated disk with an advection-dominated outer region, as the accretion rate increases, the value of $\tilde{r}$ decreases, and that $\tilde{r}$ increases with increasing $\gamma$ or decreasing $m$.

In the case where the gas pressure is dominated and the outer disk flow is still an ADAF, we obtain the temperature of the inner disk,

$$
T_{11}=\left(1+Y_{e}\right)^{-1} \frac{1}{8.315} \frac{P_{29}}{\rho_{11}}=\left(1+Y_{e}\right)^{-1} 0.874 m^{2 / 3} \tilde{\rho}_{11}^{-2 / 3} \dot{m}_{d}^{2 / 3} \tilde{f}^{2 / 3} \alpha_{-1}^{-2 / 3} \tilde{r}_{6}^{-1} r_{6}^{-1}
$$

Similarly, from equations (17) and (26), we have

$$
\int_{r_{*}}^{\tilde{r}} Q_{\nu}^{-} 2 \pi r d r=10^{51} \times \int 45.0 \tilde{\rho}_{11}^{-10 / 3}\left(1+Y_{e}\right)^{-6} m^{23 / 6} \dot{m}_{d}^{13 / 3} \tilde{f}^{13 / 3} \tilde{r}_{6}^{\left(\frac{1}{\gamma-1}-\frac{13}{2}\right)} r_{6}^{-\left(4+\frac{1}{\gamma-1}\right)} d r_{6}
$$

The neutrino luminosity of the inner disk reads

$$
\begin{aligned}
L_{\nu}= & 3.53 \times 10^{53} \operatorname{ergs~}^{-1}\left(\frac{\gamma-1}{3 \gamma-2}\right)\left(1+Y_{e}\right)^{-26 / 11} \\
& \times m^{51 / 22} \dot{m}_{d}^{21 / 11} \tilde{f}^{\frac{31}{11}} \alpha_{-1}^{-21 / 11} \tilde{r}_{6}^{\left(\frac{1}{\gamma-1}-\frac{3}{22}\right)}\left(r_{*, 6}^{\frac{2-3 \gamma}{\gamma-1}}-\tilde{r}_{6}^{\frac{2-3 \gamma}{\gamma-1}}\right) .
\end{aligned}
$$

The energy-conservation equation of the inner disk is

$$
\begin{aligned}
& \left(1-\sqrt{\frac{r_{*, 6}}{\tilde{r}_{6}}}\right)^{-31 / 11} \tilde{r}_{6}^{\left(\frac{-1}{\gamma-1}+\frac{3}{22}\right)}\left(r_{*, 6}^{\frac{2-3 \gamma}{\gamma-1}}-\tilde{r}_{6}^{\frac{2-3 \gamma}{\gamma-1}}\right)^{-1} \\
= & \frac{382(\gamma-1)}{3 \gamma-2}\left(1+Y_{e}\right)^{-26 / 11} m^{29 / 22} \dot{m}_{d}^{10 / 11} \alpha_{-1}^{-21 / 11} r_{*, 6} .
\end{aligned}
$$

From equation (45), we see that the size of the inner disk $(\tilde{r})$ also decreases with increasing the accretion rate $\dot{m}_{d}$. Table 4 gives solutions of equation (45) with different sets of parameters. We can see that $\tilde{r}$ also decrease with increasing $\tilde{\gamma}, m$ or decreasing $Y_{e}$.

We above study the case where the outer disk is advection-dominated, and find that the size of the inner disk always increase with the accretion rate. In the case where the outer disk is mainly neutrino-dominated, using expression (30) and equation (17), we obtain an energy-conservation equation in the inner disk,

$$
2.77\left(\frac{\gamma-1}{3 \gamma-2}\right) \tilde{r}_{6}^{\frac{2 \gamma-1}{\gamma-1}} \tilde{f}\left(r_{*, 6}^{\frac{2-3 \gamma}{\gamma-1}}-\tilde{r}_{6}^{\frac{2-3 \gamma}{\gamma-1}}\right)=0.924\left\{\frac{1}{r_{*, 6}}-\frac{3 \bar{f}_{\nu}}{\tilde{r}_{6}}\left[1-\frac{2}{3}\left(\frac{r_{*, 6}}{\tilde{r}_{6}}\right)^{1 / 2}\right]\right\}
$$


This equation shows us that $\tilde{r}_{6}$ in NDAF is independent of $Y_{e}, m, \dot{m}_{d}$, and $\alpha$, but only dependent on $\gamma$ and $\bar{f}_{\nu}$. The size of the inner disk is constant no matter how much the components, the accretion rate of the disk, and the mass of the central neutron star are. If the outer disk is mainly an NDAF, we have $\bar{f}_{\nu} \sim 1$. We choose several different sets of parameters to obtain the solution of equation (46) (see Table 5). As $\bar{f}_{\nu}$ increases, the value of $\tilde{r}$ decreases slightly. Here we also consider an intermediate case of $\gamma$ between $5 / 3$ and 4/3, the decline of $\gamma$ makes the size of the inner disk decrease.

However, in the above discussion about an NDAF, we have not considered the effect of neutrino opacity but simply assumed that neutrinos escape freely. Actually, if the accretion rate is sufficiently large and the disk flow is mainly an NDAF, and the disk's region near the neutron star surface can be optically thick to neutrino emission. With increasing the accretion rate, the area of this optically thick region increases. We now estimate the effect of neutrino opacity on the structure of the inner disk. Let the region of $r_{*}<r<\bar{r}$ be optically thick to neutrino emission, the region of $\bar{r}<r<\tilde{r}$ be optically thin, and the electron/positron pair capture reactions be the dominant cooling mechanism. Thus equation (17) becomes

$$
\int_{r_{*}}^{\bar{r}} \frac{4\left(\frac{7}{8} \sigma_{B} T^{4}\right)}{\tau} 2 \pi r d r+\int_{\bar{r}}^{\tilde{r}} 9 \times 10^{34} \rho_{11} T_{11}^{6} H 2 \pi r d r=\frac{3 G M \dot{M}}{4}\left\{\frac{1}{3 r_{*}}-\frac{\bar{f}_{\nu}}{\tilde{r}}\left[1-\frac{2}{3}\left(\frac{r_{*}}{\tilde{r}}\right)^{1 / 2}\right]\right\} .
$$

where we take $\varepsilon \approx 1$. Using the self-similar relations and performing some derivations, we find

$$
\begin{array}{r}
18.7\left(1+Y_{e}\right)^{8 / 5}\left(\frac{\gamma-1}{2-\gamma}\right) m^{-1 / 5} \dot{m}_{d}^{-1} \tilde{f}^{-1} \alpha_{-1}^{8 / 5} \tilde{r}_{6}^{\left(\frac{18}{5}-\frac{1}{\gamma-1}\right)}\left[\bar{r}_{6}^{-1+1 /(\gamma-1)}-r_{*, 6}^{-1+1 /(\gamma-1)}\right] \\
+2.77\left(\frac{\gamma-1}{3 \gamma-2}\right) m \dot{m}_{d} \tilde{r}_{6}^{\frac{2 \gamma-1}{\gamma-1}}\left(\bar{r}_{6}^{\frac{2-3 \gamma}{\gamma-1}}-\tilde{r}_{6}^{\frac{2-3 \gamma}{\gamma-1}}\right)=0.924 m \dot{m}_{d}\left[\frac{1}{r_{*, 6}}-\frac{3 \bar{f}_{\nu}}{\tilde{r}_{6}}+\frac{2 \bar{f}_{\nu}}{\tilde{r}_{6}}\left(\frac{r_{*, 6}}{\tilde{r}_{6}}\right)^{1 / 2}\right] .(
\end{array}
$$

The solution shown by equation (48) gives $\tilde{r}$. We take $\bar{f}_{\nu} \approx 1$ and $\alpha=0.1$. Moreover, we define a new parameter $k=\bar{r}_{6} / \tilde{r}_{6}$, and assume several sets of parameters to give the solution of equation (48).

From Table 6, we can see that the size of the inner disk increases with the accretion rate. In addition, an increase of $m$ or $k$, or an decrease of $Y_{e}$ also makes the inner-disk size larger. We will compare the analytical results from Tables 3 to 6 with numerical results in $\S 3.4$ in more details. 


\subsection{Discussion about the stellar surface boundary}

Now we want to discuss the physical condition near the neutron star surface. We know that if the rotational velocity of the neutron star surface is different from that of the inner boundary of the disk, then the disk can act a torque on the star at the stellar radius. Here we take the rotational velocity of the inner disk $\Omega \simeq \Omega_{K}$ approximately as mentioned in $\S 2.1$ and $\S 2.3$. Then we always have the stellar surface angular velocity $\Omega_{*}$ to be slower than that of the inner disk $\Omega_{K}$ (i.e., $\Omega_{*}<\Omega_{K}$ ). As a result, the kinetic energy of the accreted matter is released when the angular velocity of the matter decreases to the angular velocity of the neutron star surface. From the Newtonian dynamics, we obtain a differential equation,

$$
G_{*} r_{*}=\frac{d\left(I \Omega_{*}\right)}{d t},
$$

where $G_{*} r_{*}=\dot{M} r_{*}^{2}\left(\Omega_{K}-\Omega_{*}\right)$ is the torque acting on the star surface from the disk, $I$ is the moment of inertia of the star, $I=\xi M r_{*}^{2}$, with $\xi$ being a coefficient. The above equation can be further written as

$$
\left[\Omega_{K}-\Omega_{*}(1+\xi)\right] d M=\xi M d \Omega_{*} .
$$

From equation (50) we can see that if $\Omega_{*, 0}<\Omega_{K} /(1+\xi)$ initially, we always have $d \Omega_{*} / d M>0$, i.e., the neutron star is spun up as accretion proceeds. On the other hand, if $\Omega_{*, 0}>\Omega_{K} /(1+\xi)$ initially, then the star is always spun down by the disk. The limit value of $\Omega_{*}$ is $\Omega_{K} /(1+\xi)$ in both cases. The solution of equation (50) is

$$
\left|\Omega_{*}-\frac{\Omega_{K}}{1+\xi}\right|=\left(\frac{M_{0}}{M_{0}+\Delta M}\right)^{\frac{\xi+1}{\xi}}\left|\Omega_{*, 0}-\frac{\Omega_{K}}{1+\xi}\right| \approx\left[1-\left(\frac{\xi+1}{\xi}\right) \frac{\Delta M}{M_{0}}\right]\left|\Omega_{*, 0}-\frac{\Omega_{K}}{1+\xi}\right|,
$$

where $M_{0}$ is the initial mass of the neutron star, and $\Delta M$ is the mass of the accreted matter from the disk by the star. The change of $\Omega_{*}$ depends on the ratio $\Delta M / M_{0}$. For example, if we assume $M_{0}=1.4 M_{\odot}, \Delta M=0.01 M_{\odot}$ and set $\xi=2 / 5$, then we have $\left(\frac{\Omega_{K}}{1+\xi}-\Omega_{*}\right)=0.98\left(\frac{\Omega_{K}}{1+\xi}-\Omega_{*, 0}\right)$. Or if we take $\Delta M=0.1 M_{\odot}, \xi=2 / 5$, and the central stellar mass is unchanged, then we obtain $\left(\frac{\Omega_{K}}{1+\xi}-\Omega_{*}\right)=0.79\left(\frac{\Omega_{K}}{1+\xi}-\Omega_{*, 0}\right)$.

Here we consider that the energy released from the surface boundary is also taken away by neutrino emission, and assume that neutrinos emitted around the stellar surface are opaque. We can estimate the temperature of the neutron star surface through $\frac{7}{8} \sigma_{B} T^{4} 4 \pi r_{*} H \sim \frac{G M \dot{M}}{2 r_{*}}(2-\varepsilon)$, where $H$ is the half thickness of the inner boundary of the disk, and the parameter $\varepsilon$ has the same meaning as that in $\S 2.3$. Then we can estimate the surface temperature as $T_{11} \sim 0.415(2-\varepsilon)^{1 / 4} m^{1 / 4} \dot{m}_{d}^{1 / 4} H_{6}^{-1 / 4} r_{*, 6}^{-1 / 2}$. This estimation of the

temperature is only valid if the inner disk is optically thin for neutrinos and only the surface boundary is optically thick. If the inner disk, due to a large accretion rate, becomes optically thick to neutrino emission, the surface temperature should be higher. 


\subsection{Comparison with numerical results}

In order to give an analytical solution of the accretion disk around a neutron star in the simple model, we can choose the dominant terms in equations (18) and (19). We now consider one type of pressure to be dominated, and assume extreme cases from ADAF to NDAF. For example, we consider the neutrino-dominated region with $\bar{f}_{\nu} \sim 1$ and the advection-dominated region with $\bar{f}_{\nu}=0$. In this subsection, we solve the hyperaccretiondisk structure numerically based on the simple model. In order to compare the numerical results with what we have obtained analytically, we keep on with using the equations and definitions of all the parameters in this section. However, we first need to point out several approximations and some differences between numerical and analytical methods based on the simple model.

First of all, we choose the range of $r_{6}$ from 1 to 15 in our numerical calculations. In other words, we take the range of the accretion disk to be from the surface of the neutron star to the radius of $150 \mathrm{~km}$ as the outer boundary. During the compact-star merger or massive-star collapse, the torus around a neutron star has only some part that owns a large angular momentum to form a debris disk, so the mass of the disk may be smaller than the total mass of the torus. From view of simulations (Lee \& Ramirez-Ruiz 2007), if the debris disk forms through the merger of two neutron stars, its outer radius can be slightly smaller than the value we give above. On the other hand, the disk size may be slightly larger than that we assume above if the disk forms during the collapse of a massive star. The changes of physical variables of the disk due to a change of the outer radius may be insignificant, and we do not discuss the effect of the outer radius in this paper.

Second, we fix the viscosity parameter $\alpha$ to be 0.1 . If $\alpha$ decreases (or increases), the variables of the disk increases (or decreases). More information can be seen in analytical solutions in $\S 3.1$ and $\S 3.2$. In numerical calculations, we take $\alpha$ to be a constant.

Third, we still set $Y_{e}$ as a parameter in numerical calculations in $\S 3.4$. In order to show results clearly, we consider two conditions: one is an extreme condition with $Y_{e}=1$, which means that the disk is made mainly of electrons and protons but no neutrons; the other is $Y_{e}=1 / 9$, which means that the number ratio of electrons, protons and neutrons is $1: 1: 8$. An elaborate work should consider the effect of $\beta$-equilibrium, and we will discuss it in detail in $\S 4$.

For numerical calculations, we consider all the terms of the pressure and an intermediate case between ADAF and NDAF. In analytical calculations we take the adiabatic index $\gamma$ of the inner disk to be $5 / 3$ if the disk is gas pressure-dominated or $\gamma=4 / 3$ if the disk is radiation or degeneracy pressure-dominated. In numerical calculations, however, it is convenient to 
introduce an "equivalent" adiabatic index $\gamma$ based on the original definition of $\gamma$ from the first law of thermodynamics, $\gamma=1+P / u$, where $P$ is the pressure of the disk at a given $r$, and $u$ is the internal energy density at the same radius. Therefore $\gamma$ is a variable as a function of radius. We obtain the self-similar structure,

$$
\frac{\rho(r)}{\rho(r+d r)}=\left(\frac{r}{r+d r}\right)^{-1 /(\gamma(r)-1)}, \frac{P(r)}{P(r+d r)}=\left(\frac{r}{r+d r}\right)^{-\gamma(r) /(\gamma(r)-1)} .
$$

If $\gamma$ does not vary significantly in the inner disk, the difference between the approximate solution where $\gamma$ is a constant and the numerical solution where we introduce an "equivalent" $\gamma$ is not obvious.

In addition, some region of the accretion disk is optically thick to neutrino emission when $\dot{m}_{d}$ is sufficiently large. We use the same expressions of neutrino optical depth and emission at the beginning of $\S 3$. We also require that the neutrino emission luminosity per unit area is continuous when the optical depth crosses $\tau=1$.

We first calculate the value of $\tilde{r}$, which is the radius of the boundary layer between the inner and outer disks. Figure 1 shows $\tilde{r}$ as a function of $\dot{m}_{d}$ for different values of $m$. We can see that when the disk flow is an ADAF at a low accretion rate, $\tilde{r}$ decreases monotonously as the accretion rate increases until the value of $\tilde{r}$ reaches a minimum. At this minimum, the outer disk flow is an NDAF and most of the neutrinos generated from the outer disk can escape freely, and the effect of neutrino opacity is not important. If the accretion rate is higher and makes the effect of neutrino opacity significant, the value of $\tilde{r}$ increases with increasing the accretion rate. From Figure 1, we see that when the accretion rate is either low or sufficiently high, $\tilde{r}$ is very large and even reaches the value of the outer radius, which means that the inner disk totally covers the outer disk. In this situation, since the outer disk is covered, no part of the disk is similar to that of the accretion disk around a black hole as we discussed in $§ 3.1$, and thus we say that the entire disk becomes a self-similar structure and that the physical variables of the entire disk are adjusted to build an energy balance between heating and neutrino cooling. For this situation, we do not want to discuss in details any more. We focus on the accretion rate which allows the two-steady parts of disks to exist.

In Figure 2, we choose several special conditions to plot the density, temperature and pressure of the whole disk as functions of radius $r$. Particularly, we take $\rho, P$ and $T$ to be continuous at the boundary between the inner and outer disks. If the parameter $\dot{m}_{d}$ is larger, or the disk contains more neutrons (i.e., $Y_{e}$ becomes smaller), then the density, temperature and pressure of the disk are larger, and the change of the density and pressure is more dramatic than that of the temperature. The change of the temperature cannot be very large because it greatly affects the neutrino cooling rate of the disk. 
If the mass of the central neutron star $(m)$ becomes larger or the electron fraction $Y_{e}$ becomes smaller, then the value of $\tilde{r}$ in the monotonously decreasing segment of $\dot{m}_{d}-\tilde{r}$ becomes smaller, and the value of $\tilde{r}$ in the monotonously increasing segment of $\dot{m}_{d}-\tilde{r}$ is larger. And the minimum value of $\tilde{r}$ is almost independent of $Y_{e}$ and $m$. All of these conclusions are consistent with the analytical solutions, except for the case of the advection-dominated outer disk with the radiation pressure to be dominated. In $§ 3.2 .2$, we found that the size of the inner disk increases with increasing $m$ for our analytical solutions. However, by calculating the "equivalent" adiabatic index $\gamma$, we find that $\gamma$ decreases slightly with increasing $m$. This makes the value of $\tilde{r}_{6}$ decrease, which is also consistent with the analytical results (see Table 3). Figure 3 shows the "equivalent" adiabatic index $\gamma$ as a function of radius of the entire disk for several different sets of parameters. In the case where the accretion rate is low, the radiation pressure is important. As the accretion rate increases, the gas pressure becomes more dominant and the value of $\gamma$ is larger. On the other hand, the ratio of the degeneracy pressure to the total pressure is larger in the case of a higher accretion rate and $Y_{e} \sim 1$. However, the gas pressure is always dominant for the accretion rate chosen here. We see from Figure 3 that the change of $\gamma$ in the inner disk is insignificant.

Figure 4 shows the ratio of the radial velocity $v_{r}$ and local speed of sound $c_{s}$ as a function of $r$. The ratio is always much smaller than unity, which means that the accretion flow is always subsonic and no stalled shock exists in the disk. In many cases the peak of this ratio is just at the boundary between the inner and outer disks. The reason can be found in $\S 3.2 .1$, where we gave the analytical expression of the ratio. $f=1-\left(r_{*} / r\right)^{1 / 2}$ is the major factor that affects the value of $v_{r} / c_{s}$ of the outer disk. However, in the inner disk, $v_{r} / c_{s}$ always decreases since the isothermal sound speed can be greater at smaller radii (i.e., $c_{s} \propto r^{-1 / 2}$ ), and the radial velocity of the accreting gas, which satisfies the self-similar solution (12), cannot change dramatically for the disk matter to strike the neutron star surface.

Figure 5 shows the total neutrino emission luminosity of the entire disk around a neutron star as a function of accretion rate for parameters $M$ and $Y_{e}$ (where we do not consider neutrino emission from the stellar surface discussed in §3.3), and we compare it with the neutrino luminosity from a black-hole disk. Also, we calculate the total neutrino luminosity from the inner and outer disks. Here we roughly take the mass of the black hole to be the same as that of the neutron star, and the innermost stable circular orbit of the disk has a radius which is equal to the radius of the neutron star. We approximately use the Newtonian dynamics for simplicity. In Fig. 5, we find that the difference in neutrino luminosity between the neutron-star and black-hole cases is a strong function of the accretion rate. When the accretion rate is low $\left(\dot{m}_{d} \leq 10\right)$, the total neutrino luminosity of the black-hole disk $L_{\nu, \mathrm{BH}}$ is much smaller than that of the neutron-star disk $L_{\nu, \mathrm{NS}}$, but $L_{\nu, \mathrm{BH}}$ and $L_{\nu, \mathrm{NS}}$ are similar for a moderate accretion rate $\left(\dot{m}_{d}\right.$ from 10 to 100). Actually, this result is consistent with 
the general scenario introduced in $§ 2.1$ and the basic result shown in Fig. 1: for a low accretion rate, the black-hole disk is mainly advection-dominated with most of the viscous dissipation-driven energy to be advected into the event horizon of the black hole, and we have $L_{\nu, \mathrm{BH}} \ll G M \dot{M} /(4 r)$. On the other hand, a large size of the inner disk of the neutron-star disk for a low accretion rate makes the neutrino emission efficiency be much higher than its black-hole counterpart. However, for a moderate accretion rate, the black-hole disk is similar to the neutron-star disk, which owns a quite small inner disk, and we have $L_{\nu, \mathrm{BH}} \sim L_{\nu, \mathrm{NS}}$. Moreover, neutrino opacity leads the value of $L_{\nu, \mathrm{BH}}$ to be less again compared with $L_{\nu, \mathrm{NS}}$ for a high accretion rate, as this opacity decreases the neutrino emission efficiency in the blackhole disk but increases the size of the neutron-star disk again to balance the heat energy release.

\section{An Elaborate Model of the Disk}

In the last section we first studied the disk structure analytically. To do this, we used several approximations. First of all, we took the pressure as a summation of several extreme contributions such as the gas pressure of nucleons and electrons, and the radiation pressure of a plasma of photons and $e^{+} e^{-}$pairs. However, electrons may actually be degenerate or partially degenerate, and the neutrino pressure should also be added to the total pressure. Following Kohri et al. (2005), a lot of works about hyperaccretion disks used the FermiDirac distribution to calculate the pressure of electrons and even the pressure of nucleons. Second, neutrino cooling we used in the last section is simplified, following Popham et al. (1999) and Narayan et al. (2001) and neglecting the effect of electron degeneracy and the effect of different types of neutrinos and their different optical depth. In fact, these effects may be significant in some cases. Third, we took the electron-nucleon-radio $Y_{e}$ as a constant parameter in our analytic model in $\S 3$. Realistically, $Y_{e}$ should be calculated based on $\beta$-equilibrium and neutronization in hyperaccretion disks. In this section, we still use the assumption of outer and inner disks discussed in $\S 2$, but consider a state-of-the-art model with lots of elaborate (more physical) considerations on the thermodynamics and microphysics in the disk, which was recently developed in studying the neutrino-cooled disk of a black hole. In addition, we compare results from this elaborate model with those of the simple model discussed in $\S 3$. 


\subsection{Thermodynamics and microphysics}

The total pressure in the disk can be written as: $P=P_{\text {nuc }}+P_{\mathrm{rad}}+P_{e}+P_{\nu}$. We still consider all the nucleons to be free $\left(X_{\text {nuc }} \approx 1\right)$ as mentioned in $\S 2.2$, and ignore the photodisintegration process. Also, we replace the term of radiation pressure $11 a T^{4} / 12$ in the simple model by $a T^{4} / 3$ in this section, because the pressure of $e^{+} e^{-}$pairs can be calculated in the electron pressure $P_{e}$ with the Fermi-Dirac distribution:

$$
P_{e^{ \pm}}=\frac{1}{3} \frac{m_{e}^{4} c^{5}}{\pi^{2} \hbar^{3}} \int_{0}^{\infty} \frac{x^{4}}{\sqrt{x^{2}+1}} \frac{d x}{e^{\left(m_{e} c^{2} \sqrt{x^{2}+1} \mp \mu_{e}\right) / k_{B} T}+1},
$$

where $x=p / m_{e} c$ is the dimensionless momentum of an electron and $\mu_{e}$ is the chemical potential of the electron gas. $P_{e}$ is the summation of $P_{e^{-}}$and $P_{e^{+}}$. In addition, we take the neutrino pressure to be

$$
P_{\nu}=u_{\nu} / 3
$$

where $u_{\nu}$ is the energy density of neutrinos.

The "equivalent" adiabatic index can be expressed by

$$
\gamma=1+\left(P_{\mathrm{nuc}}+P_{\mathrm{rad}}+P_{e}+P_{\nu}\right) /\left(u_{\mathrm{nuc}}+u_{\mathrm{rad}}+u_{e}+u_{\nu}\right) .
$$

with the inner energy density to be

$$
\begin{gathered}
u_{\text {gas }}=\frac{3}{2} P_{\text {gas }}, \\
u_{\text {rad }}=3 P_{\text {rad }}, \\
u_{e^{ \pm}}=\frac{m_{e}^{4} c^{5}}{\pi^{2} \hbar^{3}} \int_{0}^{\infty} \frac{x^{2} \sqrt{x^{2}+1}}{e^{\left(m_{e} c^{2} \sqrt{x^{2}+1} \mp \mu_{e}\right) / k_{B} T}+1} d x .
\end{gathered}
$$

We then use equation (52) to obtain the self-similar inner disk.

In addition, we add the equation of charge neutrality among protons, electrons and positrons to estabilish the relation between $\rho, Y_{e}$ and $\mu_{e}$.

$$
n_{p}=\frac{\rho Y_{e}}{m_{B}}=n_{e^{-}}-n_{e^{+}},
$$

where we use the Fermi-Dirac form to calculate $n_{e^{-}}$and $n_{e^{+}}$.

Moreover, in the elaborate model, we adopt the improved formula of the neutrino cooling rate $Q_{\nu}^{-}$, the inner energy density of neutrinos $u_{\nu}$, as well as the absorption and scattering optical depth for three types neutrinos $\tau_{a, \nu_{i}(e, \mu, \tau)}$ and $\tau_{s, \nu_{i}(e, \mu, \tau)}$ following a series of previous work (e.g., Popham \& Narayan 1995, Di Matteo et al. 2002, Kohri et al. 2005, Gu et al. 
2006, Janiuk et al. 2007 and Liu et al. 2007). The three types of neutrino cooling rate per unit volume are

$$
\begin{gathered}
\dot{q}_{\nu_{e}}=\dot{q}_{\mathrm{eN}}+\dot{q}_{e^{-} e^{+} \rightarrow \nu_{e} \bar{\nu}_{e}}+\dot{q}_{\mathrm{brem}}+\dot{q}_{\mathrm{plasmon}}, \\
\dot{q}_{\nu_{\mu}}=\dot{q}_{\nu_{\tau}}=\dot{q}_{e^{-} e^{+} \rightarrow \nu_{\tau} \bar{\nu}_{\tau}}+\dot{q}_{\mathrm{brem}},
\end{gathered}
$$

where the meanings of four terms $\dot{q}_{\mathrm{eN}}, \dot{q}_{e^{-} e^{+} \rightarrow \nu_{i} \bar{\nu}_{i}}, \dot{q}_{\text {brem }}$, and $\dot{q}_{\text {plasmon }}$ have been shown at the beginning of $\S 3$. Here $\dot{q}_{\mathrm{eN}}$ and $\dot{q}_{\text {plasmon }}$ are only related to $\dot{q}_{\nu_{e}}$. Moreover, $\dot{q}_{\mathrm{eN}}$ is a summation of three terms,

$$
\dot{q}_{\mathrm{eN}}=\dot{q}_{p+e^{-} \rightarrow n+\nu_{e}}+\dot{q}_{n+e^{+} \rightarrow p+\bar{\nu}_{e}}+\dot{q}_{n \rightarrow p+e^{-}+\bar{\nu}_{e}} .
$$

The formulae of three terms in equation (62) are the same as Kohri et al. (2005), Janiuk et al. (2007) and Liu et al. (2007), who considered the effect of electron degeneracy. In addition, we use the same formulae of $\dot{q}_{e^{-} e^{+} \rightarrow \nu_{i} \bar{\nu}_{i}}, \dot{q}_{\text {brem }}$, and $\dot{q}_{\text {plasmon }}$ as the early works such as Kohri et al. (2002, 2005) and Liu et al. (2007).

Finally, different from the simple model in $\S 3$ in which we took the electron fraction $Y_{e}$ as a free parameter, in this section we calculate $Y_{e}$ by considering the $\beta$-equilibrium in the disk among electrons and nucleons following Lee et al. (2005) and Liu et al. (2007)

$$
\ln \left(\frac{n_{n}}{n_{p}}\right)=f\left(\tau_{\nu}\right) \frac{2 \mu_{e}-Q}{k_{B} T}+\left[1-f\left(\tau_{\nu}\right)\right] \frac{\mu_{e}-Q}{k_{B} T},
$$

with the weight factor $f\left(\tau_{\nu}\right)=\exp \left(-\tau_{\nu_{e}}\right)$ and $Q=\left(m_{n}-m_{p}\right) c^{2}$. Equation (63) is a combined form to allow the transition from the neutrino-transparent limit case to the neutrino-opaque limit case of the $\beta$-equilibrium.

\subsection{Numerical results in the elaborate model}

Using equations (5), (6), (10) and (11) in $\S 2.2$ and the improved treatment in $\S 4.1$, we can solve the structure of the outer disk. Then using equations (12) and (17) in $\S 2.3$, we determine the size and the structure of the inner disk, and calculate the neutrino luminosity of the entire disk.

Figure 6a shows the size of the inner disk in the elaborate model. We still choose the mass of the central neutron star to be $M=1.4 M_{\odot}$ and $M=2.0 M_{\odot}$. From Figure 6a we can see that the size of the inner disk $\tilde{r}$ decreases with increasing the accretion rate, and reaches a minimum at $\dot{M} \sim 0.1 M_{\odot} s^{-1}$. Then the value of $\tilde{r}$ increases with increasing the accretion rate. This result is well consistent with what we have found in the simple model in $\S 3$. The physical reason for this result has been discussed in $\S 3.3 .2$ and $\S 3.4$. Figure $6 \mathrm{~b}$ shows the solution of the inner disk size both in the simple model and the elaborate model. We fix the 
central neutron star $M=1.4 M_{\odot}$. In the simple model of $\S 3$, we take $Y_{e}$ as a free parameter and plot two $\dot{m}_{d}-\tilde{r}_{6}$ lines with $Y_{e}=1$ and $Y_{e}=1 / 9$, while in the elaborate model we only plot one line since $Y_{e}$ can be directly determined through $\beta$-equilibrium in the disk. From Figure $6 \mathrm{~b}$ we conclude that the solutions of the two models are basically consistent with each other.

In Figure 7, we shows the "equivalent" adiabatic index $\gamma$ and the electron fraction $Y_{e}$ in the entire disk for three values of the accretion rate $\dot{M}=0.01 M_{\odot} s^{-1}, 0.1 M_{\odot} s^{-1}$ and $1.0 M_{\odot} s^{-1}$, and for two values of the mass of the central neutron star $M=1.4 M_{\odot}$ and $2.0 M_{\odot}$. The "equivalent" adiabatic index $\gamma$ increases with increasing the accretion rate in most region of the disks, since gas will take over electrons and radiation to be the dominant pressure when the accretion rate is high enough. In addition, $\gamma$ decreases as the radius decreases in the inner disk. These are consistent with the results of the simple model (see Fig. 3). From Figure 7b, we can see that $Y_{e} \sim 1$ when the accretion rate is low, and $Y_{e} \ll 1$ when the accretion rate becomes sufficiently high. This result is consistent with Kohri et al. (2005, their Fig. 6b). Chen \& Beloborodov (2007) and Liu et al. (2007) showed the electron fraction $Y_{e} \leq 0.5$ in the disk, since they supposed that initial neutrons and protons come from photodisintegration of $\alpha$-particles at some large radius far from a central black hole. However, since the hyperaccretion disk around a neutron star we discuss has a size smaller than that of a black-hole disk, we consider the mass fraction of free nucleons $X_{\text {nuc }}=1$ in the entire disk. Therefore, the fraction of protons can be slightly higher and it is possible that the protons are richer than neutrons in the disk or $Y_{e} \geq 0.5$ if the accretion rate is low enough.

Figure 8 and Figure 9a show the density, temperature, pressure and the neutrino luminosity per unit area in the entire disk with three different accretion rates. We fix $M=1.4 M_{\odot}$, and also plot two other curves of solutions in the simple model with $Y_{e}=1$ and $Y_{e}=1 / 9$. The density $\rho$ and pressure $P$ in the elaborate model are smaller than those in the simple model when the accretion rate is low $\left(\dot{m}_{d}=1.0\right)$, but are similar to the solution of the simple model with $Y_{e}=1 / 9$ in the high accretion rate $\left(\dot{m}_{d}=100\right)$. In addition, $\rho$ and $P$ in the elaborate model change from one solution $\left(Y_{e}=1\right)$ to another solution $\left(Y_{e}=1 / 9\right)$ in the simple model for an intermediate accretion rate (e.g., $\left.\dot{m}_{d} \sim 10\right)$, since $Y_{e} \sim 0.5$ in the outer edge of the disk and $Y_{e} \ll 1$ in the inner disk. The distribution of the neutrino cooling rate $Q_{\nu}^{-}$(luminosity per unit area) in the elaborate model is almost the same as that in the simple model with $Y_{e}=1$ and low accretion rate or $Y_{e}=1 / 9$ and a high accretion rate. However, the value of $Q_{\nu}^{-}$is still different in these two models for the region that is optically thick to neutrino emission in the disk.

We also plot the the total neutrino emission luminosity of the entire disk, the outer and 
inner disks as functions of accretion rate with the central neutron star mass of $1.4 M_{\odot}$, and compare the total neutrino luminosity with that of the black-hole disk (Figure 9b). The results are similar to what we have found in the simple model (Figure 5).

\section{Conclusions and Discussions}

In this paper we have studied the structure, energy advection and conversion, and neutrino emission of a hyperaccretion disk around a neutron star. We considered a quasisteady disk model without any outflow. Similar to the disk around a black hole, the neutron star disk with a huge mass accretion rate is extremely hot and dense, opaque to photons, and thus is only cooled via neutrino emission, or even optically thick to neutrino emission in some region of the disk if the accretion rate is sufficiently high. However, a significant difference between black hole and neutron star disks is that the heat energy of the disk can be advected into the event horizon if the central object is a black hole, but if the central object is a neutron star, the heat energy should be eventually released from a region of the disk near the stellar surface. As a result, the neutrino luminosity of the neutron star disk should be much larger than that in black hole accretion. We approximately took the disk as a Keplerian disk. According to the Virial theorem, one half of the gravitational energy in such a disk is used to heat the disk and the other half to increase the rotational kinetic energy of the disk. We assumed that most of the heat energy generated from the disk is still cooled from the disk via neutrino emission and the rotational energy is used to spin up the neutron star or is released on the stellar surface via neutrino emission.

In a certain range of hypercritical accretion rates, depending on the mechanisms of energy heating and cooling in the disk, we considered a two-region, steady-state disk model. The outer disk is similar to the outer region of a black hole disk. We used the standard viscosity assumption, Newtonian dynamics and vertically integrated method to study the structure of the outer disk. Since the radial velocity of the disk flow is always subsonic, no stalled shock exists in the disk and thus we considered that physical variables in the disk change continuously when crossing the boundary layer between the inner and outer disks. The inner disk, which expands until a heating-cooling balance is built, could satisfy a self-similar structure as shown by equation (12).

In this paper we first studied the disk structure analytically. To do this, we adopted a simple disk model based on the analytical method. We took the pressure as a summation of several extreme contributions and simple formulae of neutrino cooling. And we took the electron fraction $Y_{e}$ as a parameter in the simple model. We used an analytical method to find the dominant-pressure distribution (Table 2) and the radial distributions of the density, 
temperature and pressure (solutions 20, 21, 26, 30, 32) in the outer disk. Then we used the equation of energy balance between heating and neutrino cooling to calculate the size of the inner disk in four different cases: whether the advection-dominated outer disk is radiation or gas pressure-dominated, and whether the neutrino-cooled outer disk is optically thin or thick to neutrino emission (Table 3 to 6). Subsequently, we numerically calculated the size of the inner disk, the structure, and energy conversion and emission of the entire disk in the simple model (Fig. 2 to Fig. 5) and compared the numerical results with the analytical results. The numerical results are consistent with the analytical ones from the simple model.

When the accretion rate is sufficiently low, most of the disk is advection-dominated, the energy is advected inward to heat the inner disk, and eventually released via neutrino emission in the inner disk. In this case, the inner disk is very large, and quite different from a black hole disk, which advects most of the energy inward into the event horizon. If the accretion rate is higher, then physical variables such as the density, temperature and pressure become larger, the disk flow becomes NDAF, the advected energy becomes smaller, and heating of the inner disk becomes less significant. As a result, the size of the inner disk is much smaller, and the difference between the entire disk and the black hole disk becomes less significant. Furthermore, if the accretion rate is large enough to make neutrino emission optically thick, then the effect of neutrino opacity becomes important so that the efficiency of neutrino emission from most of the disk decreases and the size of the inner disk again increases until the entire disk becomes self-similar. Besides, a different mass of the central star or a different electron-nucleon ratio also makes physical variables and properties of the disk different. However, the accretion rate plays a more significant role in the disk structure and energy conversion, as it varies much wider than the other parameters.

The simple model is based on the early works such as Popham et al. (1999) and Narayan et al. (2001). We found that the simple model in fact gives us a clear physical picture of the hyperaccretion disk around a neutron star, even if we used some simplified formulae in thermodynamics and microphysics in the disk. In $\S 4$ we considered an elaborate model, in which we calculated the pressure of electrons and positrons by using the Fermi-Dirac distribution and replaced the factor $11 / 12$ by $1 / 3$ in the radiation-pressure equation. We adopted more advanced expressions of the neutrino cooling rates, including the effect of all three types of neutrinos and the electron degeneracy. Moreover, we considered $\beta$-equilibrium in the disk to calculate the electron fraction $Y_{e}$. Then, in the elaborate model, we also calculated the the size of the inner disk (Fig. 6), the radial distributions of the "equivalent" adiabatic index $\gamma$, the electron fraction (Fig. 7), the density, temperature and pressure in the disk (Fig. 8), the neutrino cooling rate distribution of the disk (Fig. 9a), the neutrino emission luminosity from the inner and outer disks, and the total neutrino luminosity of a neutron-star disk compared with that of a black-hole disk (Fig. 9b). 
The electron fraction $Y_{e}$ was also determined in the elaborate model. We found that $Y_{e}$ drops with increasing the accretion rate in the outer disk. $Y_{e}$ can be greater than 0.5 at a large radius if the accretion rate is sufficiently low, and $Y_{e} \ll 1$ in the disk when the accretion rate is high enough. If we put these results of $Y_{e}$ in the elaborate model into the simple model correctly (i.e. $Y_{e} \sim 1$ for low accretion rate and $Y_{e} \ll 1$ for high accretion rate), we find that they are basically consistent with each other (see Fig. 6, Fig. 7, Fig. 8, Fig. 9), and also consistent with most of the early works (see the discussion in the end of $\S 3.1)$.

A main difference in the structure between the simple and elaborate models is caused by different expressions of pressure adopted in the two models. In order to see it clearly, we introduce the third model here. We still keep $P_{\mathrm{rad}}=11 a T^{4} / 12$ as the simple model but just change the relativistic degeneracy pressure term of electrons (the second term in equation 19) to the Fermi-Dirac distribution (formula 53 for $P_{e^{-}}$), and use all the other formulae in $\S 4$. In other words, the third model is introduced by only changing one pressure term in the elaborate model. We can find that the results from the third model are even more consistent with those of the simple model than the elaborate model in $\S 4$. Take Figure 10 as an example. We compare the solution of the inner disk of the third model with that of the simple model. From Fig. 10 we can see that if the accretion rate is low and $Y_{e} \sim 1$, the thick solid line is much closer to the thin dashed line, which results from the simple model with $Y_{e}=1$; and if the accretion rate is high enough and $Y_{e} \ll 1$, the solid line is much closer to the thick dotted line, which is the result from the simple model with $Y_{e}=1 / 9$. Compared with Fig. 8a, this result is even more consistent with the simple model. The values of density $\rho$ and pressure $P$ in Fig. 10, for a low accretion rate, are smaller than those of the simple model, which is also due to different expressions of the radiation pressure used in these two models in $\S 3$ and $\S 4$. Therefore, we conclude that the main difference of the results between the simple model in $\S 3$ and the elaborate model in $\S 4$ come from different expressions of the pressure in disks. However, we believe that the pressure formulae given in the elaborate model are more realistic since $11 a T^{4} / 12$ is only an approximated formula for the pressure of a plasma of photons and $e^{+} e^{-}$pairs. On the other hand, as what has been pointed out by Lee et al. (2005) and Liu et al. (2007), formulae $P_{\mathrm{rad}}=a T^{4} / 3$ and (53) in $\S 4.1 .1$ are better and can automatically take relativistic $e^{+} e^{-}$pairs into account in the expression of $P_{e}$.

The different expression of the neutrino cooling rate $Q_{\nu}^{-}$makes the neutrino luminosity distribution different in the region where is optically thick to neutrino emission. A more advanced expression of neutrino cooling rate $Q_{\nu}^{-}$gives better results of the neutrino luminosity per unit area than that given by the rough expression $\frac{7}{8} \sigma_{B} T^{4} / \tau$ in $\S 3$.

In this paper we studied the disk without any outflow, which may exist if the disk flow 
is an ADAF. However, it is still unclear whether an outflow or neutrino cooling plays a more important role since the size of the disk is quite small. The other case that we ignored is that, if the radius of the central neutron star is smaller than that of the innermost stable circular orbit of the accretion disk, the accreting gas eventually falls onto the neutron star freely. In this case, a shock could form in the region between the innermost stable circular orbit and neutron star surface (Medvedev 2004). This effect can be studied if other effects such as the equation of state of a differentially-rotating neutron star and its mass-radius relation are together involved.

Neutrinos from a hyperaccretion disk around a neutron star will be possibly annihilated to electron/positron pairs, which could further produce a jet. It would be expected that such a jet is more energetic than that from the neutrino-cooled disk of a black hole with same mass and accretion rate as those of the neutron star (Zhang \& Dai 2008, in preparation). This could be helpful to draw the conclusion that some GRBs originate from neutrino annihilation rather than magnetic effects such as the Blandford-Znajek effect.

We considered a central neutron star with surface magnetic field weaker than $B_{s, \mathrm{cr}} \sim$ $10^{15}-10^{16} \mathrm{G}$ for typical hyperaccretion rates in this paper. For magnetars (i.e., neutron stars with ultra-strongly magnetic fields of $\sim B_{s, \mathrm{cr}}$ ), however, the magnetic fields could play a significant role in the global structure of hyperaccretion disks as well as underlying microphysical processes, e.g., the quantum effect (Landau levels) on the electron distribution and magnetic pressure in the disks could become important. Thus, the effects of an ultrastrongly magnetic field on hyperaccretion disks around neutron stars are an interesting topic, which deserves a detailed study.

\section{Acknowledgements}

We would like to thank the referees for their valuable comments that have allowed us to improve this manuscript. We also thank P. F. Chen for his helpful discussion in numerical solutions and Yefei Yuan and Bing Zhang for their useful suggestions. This work is supported by the National Natural Science Foundation of China (grants 10221001 and 10640420144) and the National Basic Research Program of China (973 program) No. 2007CB815404.

\section{REFERENCES}

Beloborodov, A. M. 2003, ApJ, 588, 931

Bethe, H. A. 1990, Rev. Mod. Phys., 62, 801 
Brown, G. E., \& Weingartner, J. C. 1994, ApJ, 436, 834

Chen, W., \& Beloborodov, A. M. 2007, ApJ, 657, 383

Chevalier, R. A. 1989, ApJ, 346, 847

Chevalier, R. A. 1996, ApJ, 459, 322

Dai, Z. G. 2004, ApJ, 606, 1000

Dai, Z. G., \& Lu, T. 1998a, A\&A, 333, L87

Dai, Z. G., \& Lu, T. 1998b, Phys. Rev. Lett., 81, 4301

Dai, Z. G., Wang, X. Y., Wu, X. F., \& Zhang, B. 2006, Science, 311, 1127

Di Matteo, T., Perna, R., \& Narayan, R. 2002, ApJ, 579, 706

Fan, Y. Z., \& Xu, D. 2006, MNRAS, 372, L19

Frank, J., King, A., \& Raine, D. 2002, Accretion Power in Astrophysics (Cambridge: Cambridge Univ. Press)

Gu, W.-M., Liu, T., \& Lu, J.-F. 2006, ApJ, 643, L87

Janiuk, A., Yuan, Y., Perna, R., \& Di Matteo, T. 2007, ApJ, 664, 1011

Kluźniak, W., \& Ruderman, M. 1998, ApJ, 505, L113

Kluźniak, W., \& Wilson J. R. 1991, ApJ, 372, L87

Kohri, K., \& Mineshige, S. 2002, ApJ, 577, 311

Kohri, K., Narayan, R., \& Piran, T. 2005, ApJ, 629, 341

Lee, W. H., \& Ramirez-Ruiz, E. 2007, arXiv: astro-ph/0701874v3

Liang, E. W., Zhang, B.-B., \& Zhang, B. 2007, ApJ, 670, 565

Liu, T., Gu, W. M., Xue, L., \& Lu, J. F. 2007, ApJ, 661, 1025

Mazzali, P. A. et al. 2006, Nature, 442, 1018

Medvedev, M. V. 2004, arXiv:astro-ph/0407062

Medvedev, M. V., \& Narayan, R. 2001, ApJ, 554, 1255 
Mészáros, P. 2006, Rep. Prog. Phys., 69, 2259

Nakar, E. 2007, Phys. Rep., 442, 166

Narayan, R, Igumenshchev, I. V., \& Abramowicz, M. A. 2000, ApJ, 539, 798

Narayan, R, Mahadevan, R., \& Quataert, E. 1998, in The Theory of Black Hole Accretion Discs, ed. M. A. Abramowicz, G. Bjornsson, \& J. E. Pringle (Cambridge: Cambridge Univ. Press)

Narayan, R., Paczynski, B., \& Piran, T. 1992, ApJ, 395, L83

Narayan, R., Piran, T., \& Kumar, P. 2001, ApJ, 557, 949

Narayan, R., \& Yi, I. 1994, ApJ, 428, L13

Paczyński, B., \& Haensel, P. 2005, MNRAS, 362, L4

Perna, R., Armitage, P. J., \& Zhang, B. 2006, ApJ, 636, L29

Piran, T. 2004, Rev. Mod. Phys., 76, 1143

Proga, D., \& Zhang, B. 2006, MNRAS, 370, L61

Popham, R., \& Narayan, R., 1995, ApJ, 442, 337

Popham, R., Woosley, S. E, \& Fryer, C. 1999, ApJ, 518, 356

Shakura, N. I., \& Sunyaev, R. A. 1973, A\&A, 24, 337

Shapiro, S. L., \& Salpeter, E. E. 1975, ApJ, 198, 671

Soderberg, A. M. et al. 2006, Nature, 442, 1014

Spruit, H. C., Matsuda, T., Inoue, M., \& Sawada, K. 1987, MNRAS, 229, 517

Wang, X. Y., Dai, Z. G., Lu, T., Wei, D. M., \& Huang, Y. F. 2000, A\&A, 357, 543

Wheeler, J. C., Yi, I., Hoflich, P., \& Wang, L. 2000, ApJ, 537, 810

Yu, Y. W., \& Dai, Z. G. 2007a, A\&A, 470, 119

Yu, Y. W., Liu, X. W., \& Dai, Z. G. 2007b, ApJ, 671, 637

Yuan, Y. F. 2005, Phys. Rev. D, 72, 013007

Zhang, B. 2007, Chin. J. Astron. Astrophys., 7, 1 
Zhang, B., \& Mészáros, P. 2001, ApJ, 552, L35

Zhang, B., \& Mészáros, P. 2004, IJMPA, 19, 2385

This preprint was prepared with the AAS IATEX macros v5.2. 


\begin{tabular}{|l|l|r|}
\hline notation & definition & $\S /$ Eq. \\
\hline$m$ & mass of the central neutron star, $m=M / 1.4 M_{\odot}$ & $\S 3.1,(20)$ \\
$\dot{m}_{d}$ & mass accretion rate, $\dot{m}_{d}=\dot{M} / 0.01 M_{\odot} \mathrm{s}^{-1}$ & $\S 3.1,(20)$ \\
$Y_{e}$ & ratio of the electron to nucleon number density in the disk & $\S 2.2,(19)$ \\
$r_{*}$ & radius of the neutron star & $\S 2.3,(13)$ \\
$r_{\text {out }}$ & outer radius of the disk & $\S 2.3,(13)$ \\
$\Omega_{*}$ & angular velocity of the stellar surface & $\S 3.3,(149)$ \\
$\varepsilon$ & efficiency of energy release in the inner disk & $\S 2.3,(17)$ \\
$\tilde{r}$ & radius between the inner and outer disks & $\S 2.3,(12)$ \\
$\bar{r}$ & radius between the neutrino optically-thick \& -thin regions \\
$k=\bar{r} / \tilde{r}$ & parameter to measure the neutrino optically-thick region & $\S 3.2,(47)$ \\
$f=1-\sqrt{\frac{r_{*}}{r}}$ & useful factor as a function of $r$ & $\S 2.2,(17)$ \\
$\tilde{f}=1-\sqrt{\frac{r_{*}}{\tilde{r}}}$ & value of $f$ at radius $\tilde{r}$ & $\S 3.2,(138)$ \\
$\bar{f}$ & average efficiency of neutrino cooling in the outer disk & $\S 2.3,(13)$ \\
\hline
\end{tabular}

Table 1: Notation and definition of some quantities in this paper.

\begin{tabular}{|c|c|c|c|c|}
\hline dominant pressure & accretion flow & range of $\dot{m}_{d}$ & $Y_{e} \sim 1$ & $Y_{e} \ll 1$ \\
\hline 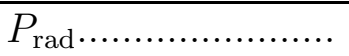 & ADAF & (a) \& (b) & 0.453 & 7.25 \\
\hline$P_{\text {gas }} \ldots$ & $\mathrm{ADAF}$ & (c) \& (d) & 1.73 & - \\
\hline$P_{\text {gas }} \ldots \ldots \ldots \ldots \ldots \ldots \ldots \ldots \ldots \ldots \ldots \ldots$ & NDAF & (e) \& (f) & 64.5 & $\infty$ \\
\hline 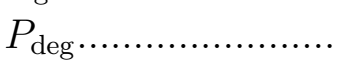 & NDAF & (g) & $\infty$ & - \\
\hline
\end{tabular}

Table 2: Range of the accretion rate in different regions. (a) $\dot{m}_{d}<0.504 m^{1 / 2} \alpha_{-1}^{5 / 3} r_{6}^{5 / 2} f^{1 / 6}$; (b) $\dot{m}_{d}<644\left(1+Y_{e}\right)^{-4} m^{7 / 2} \alpha_{-1} r_{6}^{-3 / 2} f^{7 / 2} ; \quad(\mathrm{c}) \dot{m}_{d}>644\left(1+Y_{e}\right)^{-4} m^{7 / 2} \alpha_{-1} r_{6}^{-3 / 2} f^{7 / 2}$; (d) $\dot{m}_{d}<4.81 \times 10^{-3}\left(1+Y_{e}\right)^{13 / 5} m^{-29 / 20} \alpha_{-1}^{21 / 10} r_{6}^{47 / 20} f^{-2} ; \quad(\mathrm{e}) \dot{m}_{d}>4.81 \times 10^{-3}(1+$ $\left.Y_{e}\right)^{13 / 5} m^{-29 / 20} \alpha_{-1}^{21 / 10} r_{6}^{47 / 20} f^{-2} ; \quad(\mathrm{f}) \dot{m}_{d}<0.148 Y_{e}^{-4}\left(1+Y_{e}\right)^{27 / 5} m^{-11 / 10} \alpha_{-1}^{19 / 10} r_{6}^{33 / 20} f^{-1}$; (g) $\dot{m}_{d}>0.148 Y_{e}^{-4}\left(1+Y_{e}\right)^{27 / 5} m^{-11 / 10} \alpha_{-1}^{19 / 10} r_{6}^{33 / 20} f^{-1}$.

\begin{tabular}{rcccc}
\hline \hline$\dot{m}_{d}$ & 0.01 & 0.05 & 0.1 & 0.5 \\
\hline Case 1 & 6.38 & 3.53 & 2.76 & 1.65 \\
Case 2 & 13.87 & 5.66 & 3.92 & 1.84 \\
Case 3 & 6.83 & 3.76 & 2.94 & 1.74 \\
\hline \hline
\end{tabular}

Table 3: Equation (41) gives $\tilde{r}_{6}$ in several cases. Case 1: $m=1, \gamma=4 / 3$; case $2: m=1, \gamma=$ 1.4; case $3: m=2.0 / 1.4, \gamma=4 / 3$. 


\begin{tabular}{rccccc}
\hline \hline$\dot{m}_{d}$ & 0.5 & 1 & 3 & 5 & 10 \\
\hline Case 1 & 2.17 & 1.91 & 1.62 & 1.52 & 1.42 \\
Case 2 & 2.05 & 1.83 & 1.59 & 1.50 & 1.40 \\
Case 3 & 1.68 & 1.54 & 1.38 & 1.33 & 1.27 \\
Case 4 & 1.97 & 1.76 & 1.52 & 1.44 & 1.36 \\
\hline \hline
\end{tabular}

Table 4: Equation (45) gives $\tilde{r}_{6}$ in several cases. Case 1: $Y_{e}=1, \gamma=5 / 3, m=1$; case 2: $Y_{e}=1, \gamma=3 / 2, m=1$; case $3: Y_{e}=1 / 9, \gamma=5 / 3, m=1$; case $4: Y_{e}=1, \gamma=5 / 3, m=$ 2.0/1.4.

\begin{tabular}{rccc}
\hline \hline $\bar{f}_{\nu} \backslash \gamma$ & $5 / 3$ & $3 / 2$ & $4 / 3$ \\
\hline 0.9 & 1.31 & 1.28 & 1.26 \\
0.7 & 1.46 & 1.43 & 1.39 \\
0.5 & 1.56 & 1.53 & 1.47 \\
\hline \hline
\end{tabular}

Table 5: Equation (46) gives $\tilde{r}_{6}$. We take $\bar{f}_{\nu}$ and $\gamma$ as parameters to solve this equation.

\begin{tabular}{rcccc}
\hline \hline$\dot{m}_{d}$ & 80 & 100 & 120 & 150 \\
\hline Case 1 & 4.28 & 5.13 & 5.94 & 7.10 \\
Case 2 & 6.26 & 7.48 & 8.65 & 10.33 \\
Case 3 & 5.09 & 6.09 & 7.05 & 8.43 \\
Case 4 & 4.10 & 4.99 & 5.86 & 7.11 \\
Case 5 & 4.87 & 5.90 & 6.89 & 8.32 \\
\hline \hline
\end{tabular}

Table 6: Equation (48) gives $\tilde{r}_{6}$ in several different cases. Case 1: $Y_{e}=1, m=1, k=1, \gamma=$ 5/3; case $2: Y_{e}=1 / 9, m=1, k=1, \gamma=5 / 3$; case $3: Y_{e}=1, m=2.0 / 1.4, k=1, \gamma=5 / 3$, Case 4: $Y_{e}=1, m=1, k=0.7, \gamma=5 / 3$; case $5: Y_{e}=1, m=1, k=1, \gamma=3 / 2$. 


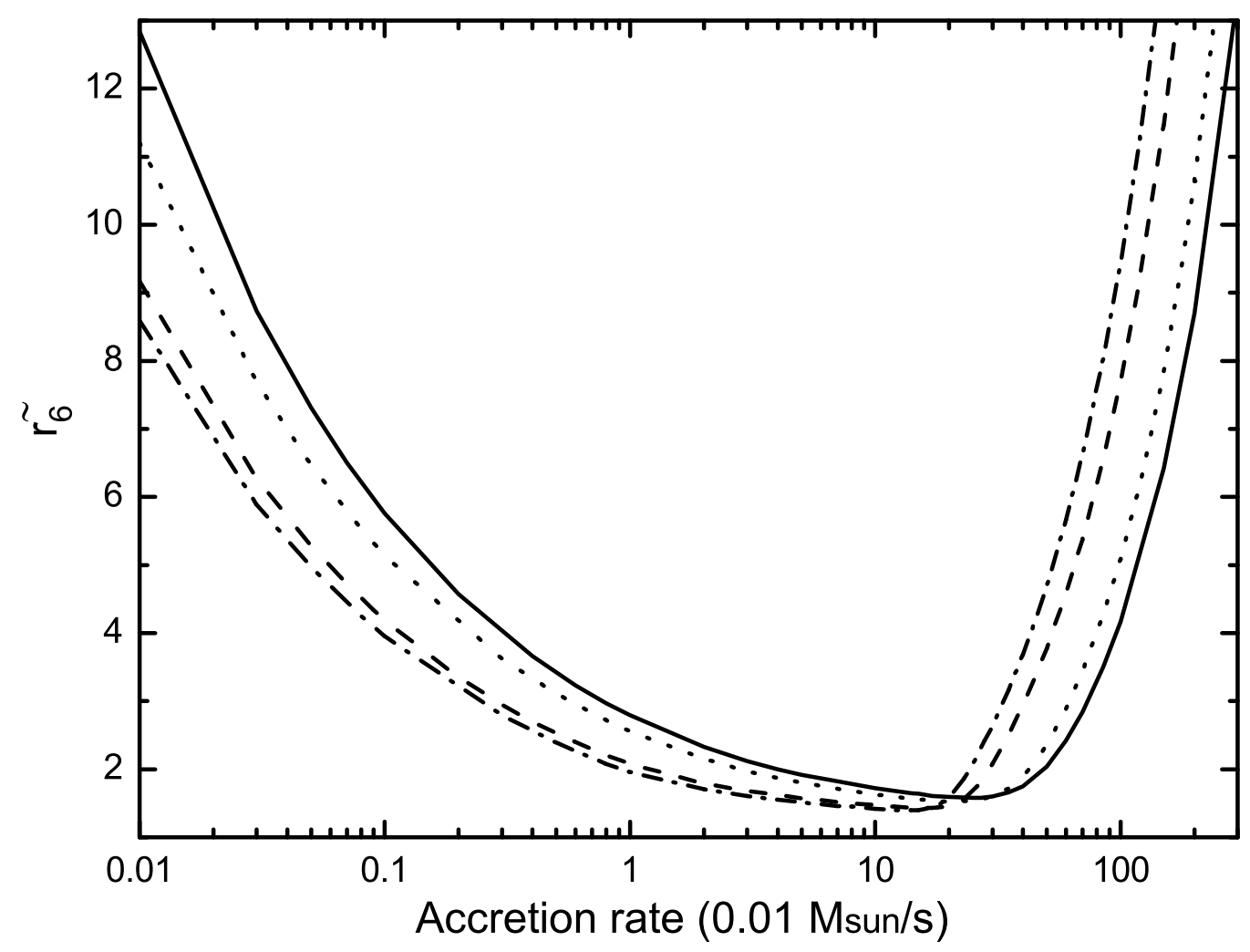

Fig. 1. - The radius $\tilde{r}_{6}$ of the boundary layer between the inner and outer disks as a function of accretion rate $\dot{m}_{d}$ in the simple model for several sets of parameters: (1) $M=1.4 M_{\odot}$ and $Y_{e}=1.0$ (solid line), (2) $M=1.4 M_{\odot}$ and $Y_{e}=1 / 9$ (dashed line), (3) $M=2.0 M_{\odot}$ and $Y_{e}=1.0$ (dotted line), and (4) $M=2.0 M_{\odot}$ and $Y_{e}=1 / 9$ (dash-dotted line). 

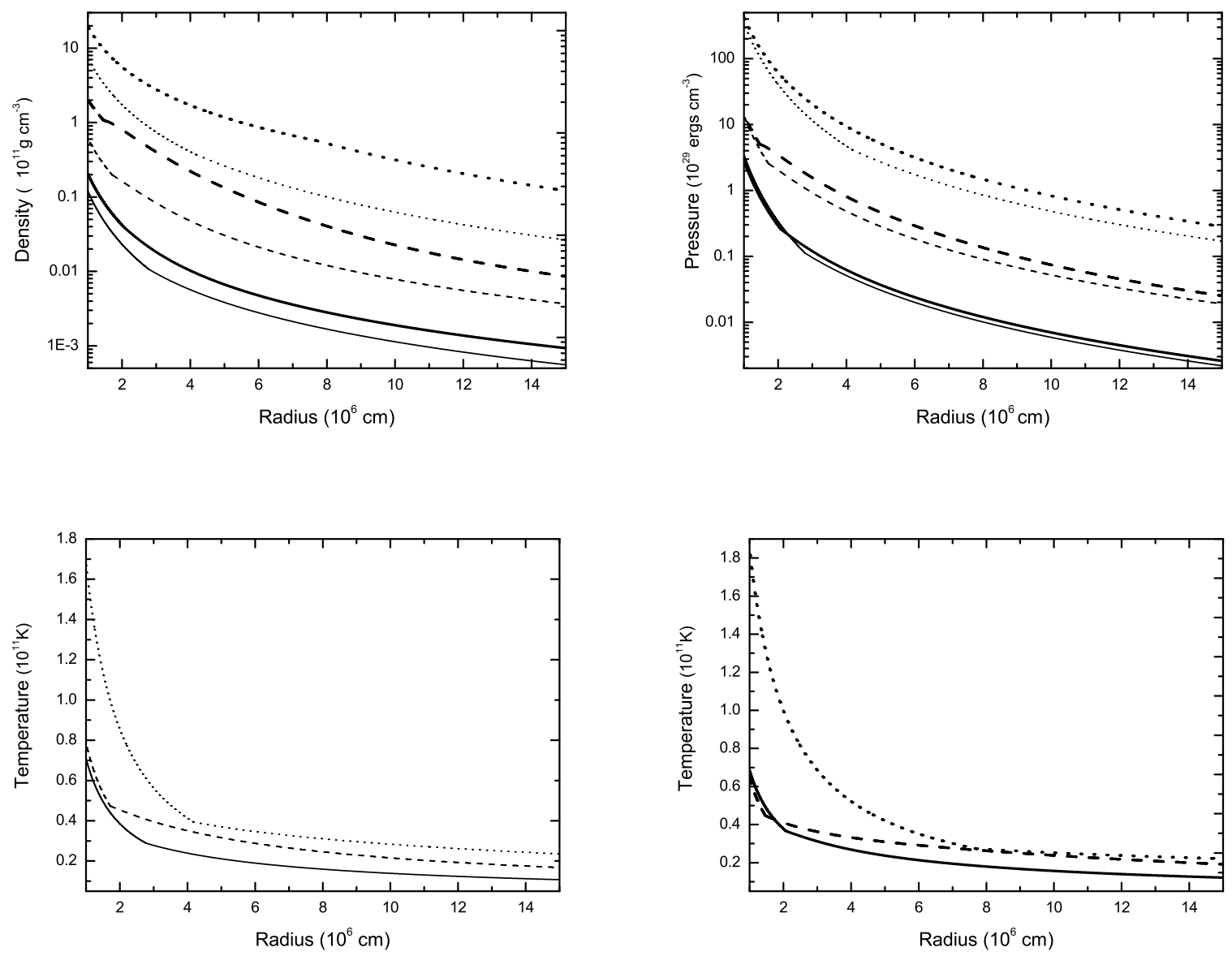

Fig. 2.- The density (in units of $10^{11} \mathrm{~g} \mathrm{~cm}^{-3}$ ), pressure (in units of $10^{11} \mathrm{ergs}^{-3}$ ) and temperature (in units of $10^{11} \mathrm{~K}$ ) of the disk as functions of radius $r$ (in units of $10^{6} \mathrm{~cm}$ ) in the simple model for several sets of parameters: (1) $M=1.4 M_{\odot}, Y_{e}=1.0$, and $\dot{M}=0.01 M_{\odot} \mathrm{s}^{-1}$ (thin solid line), (2) $M=1.4 M_{\odot}, Y_{e}=1.0$, and $\dot{M}=0.1 M_{\odot} \mathrm{s}^{-1}$ (thin dashed line), (3) $M=1.4 M_{\odot}, Y_{e}=1.0$, and $\dot{M}=1.0 M_{\odot} \mathrm{s}^{-1}$ (thin dotted line), (4) $M=2.0 M_{\odot}, Y_{e}=1.0$, and $\dot{M}=0.01 M_{\odot} \mathrm{s}^{-1}$ (thick solid line), (5) $M=2.0 M_{\odot}, Y_{e}=1.0$, and $\dot{M}=0.1 M_{\odot} \mathrm{s}^{-1}$ (thick dashed line), and (6) $M=2.0 M_{\odot}, Y_{e}=1.0$, and $\dot{M}=1.0 M_{\odot} \mathrm{s}^{-1}$ (thick dotted line). 


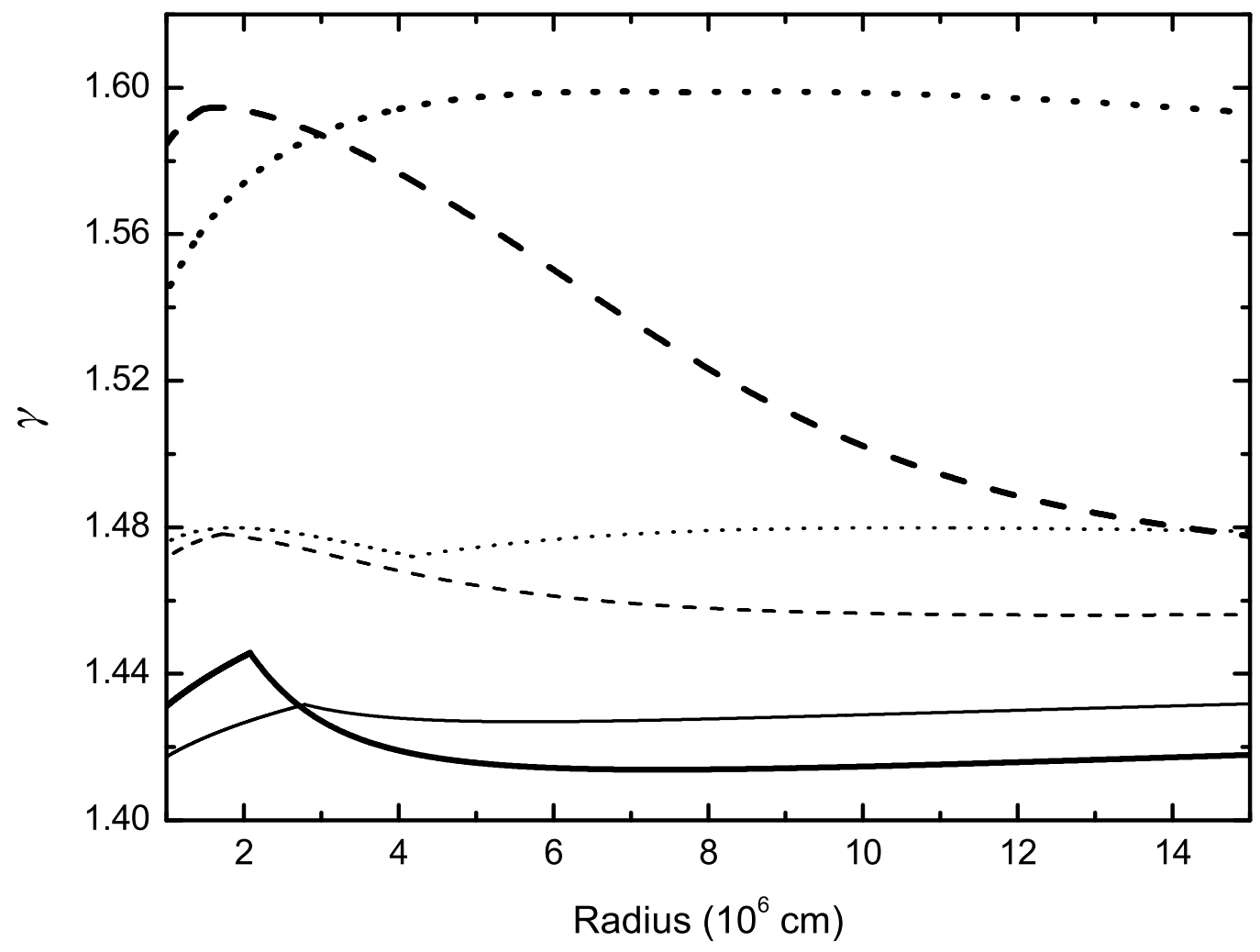

Fig. 3.- The "equivalent" adiabatic index $\gamma$ of the disk as a function of radius $r$ in the simple model. The meanings of different lines are the same as those in Fig. 2. 

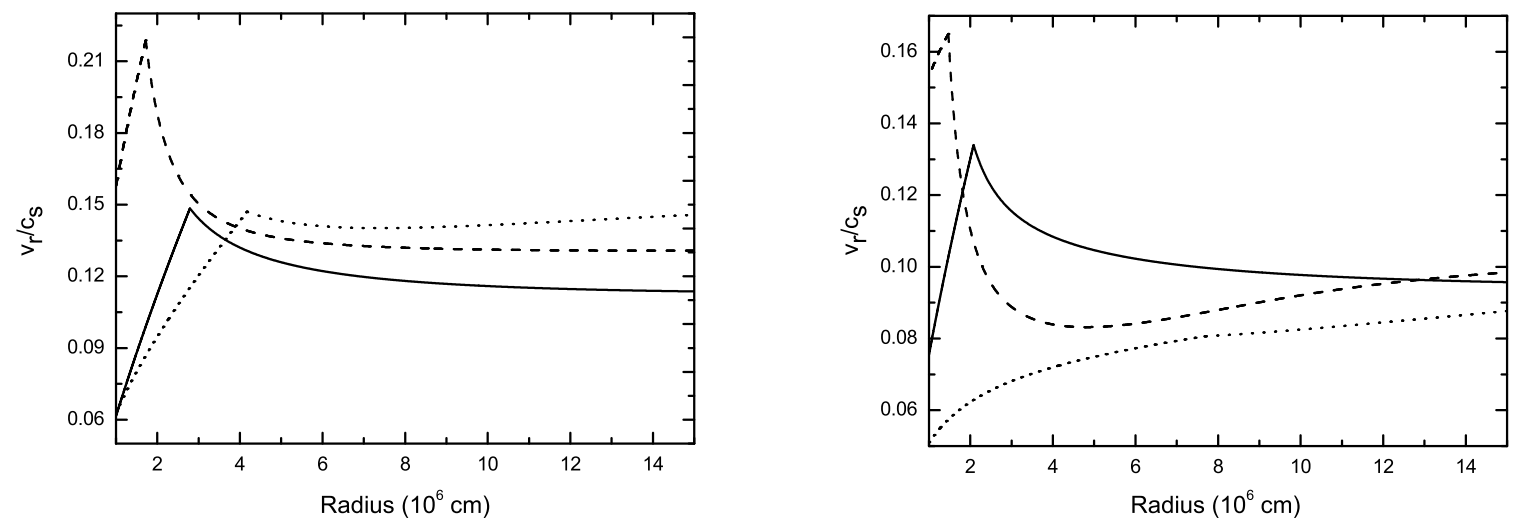

Fig. 4. - The ratio of the radial velocity $v_{r}$ and local speed of sound $c_{s}$ as a function of $r$. (a) Left panel: $M=1.4 M_{\odot}, Y_{e}=1.0$; (b) Right panel: $M=1.4 M_{\odot}, Y_{e}=1 / 9$. The accretion rate: $\dot{m}_{d}=0.01 M_{\odot} \mathrm{s}^{-1}($ solid line $), \dot{m}_{d}=0.1 M_{\odot} \mathrm{s}^{-1}($ dashed line $)$, and $\dot{m}_{d}=1.0 M_{\odot} \mathrm{s}^{-1}$ (dotted line)
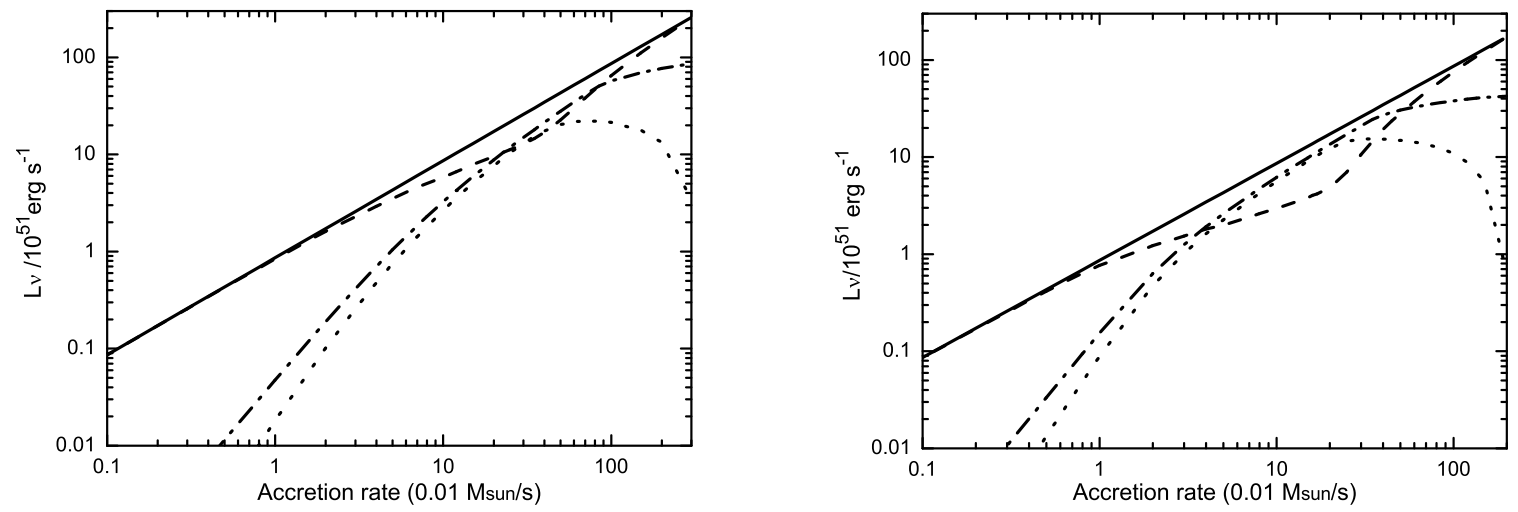

Fig. 5.- Neutrino luminosity from the disk around a neutron star in the simple model. $L_{\nu}$ is in units of $10^{51} \mathrm{ergs} \mathrm{s}^{-1}$. (a) Left panel: $M=1.4 M_{\odot}$ and $Y_{e}=1.0$. (b) Right panel: $M=1.4 M_{\odot}$ and $Y_{e}=1 / 9$. The solid line corresponds to the maximum energy release rate of the disk around a neutron star, the dashed line to the neutrino luminosity from the inner disk, the dotted line to the neutrino luminosity from the outer disk, and the the dash-dotted line to the neutrino luminosity from a black hole disk. 

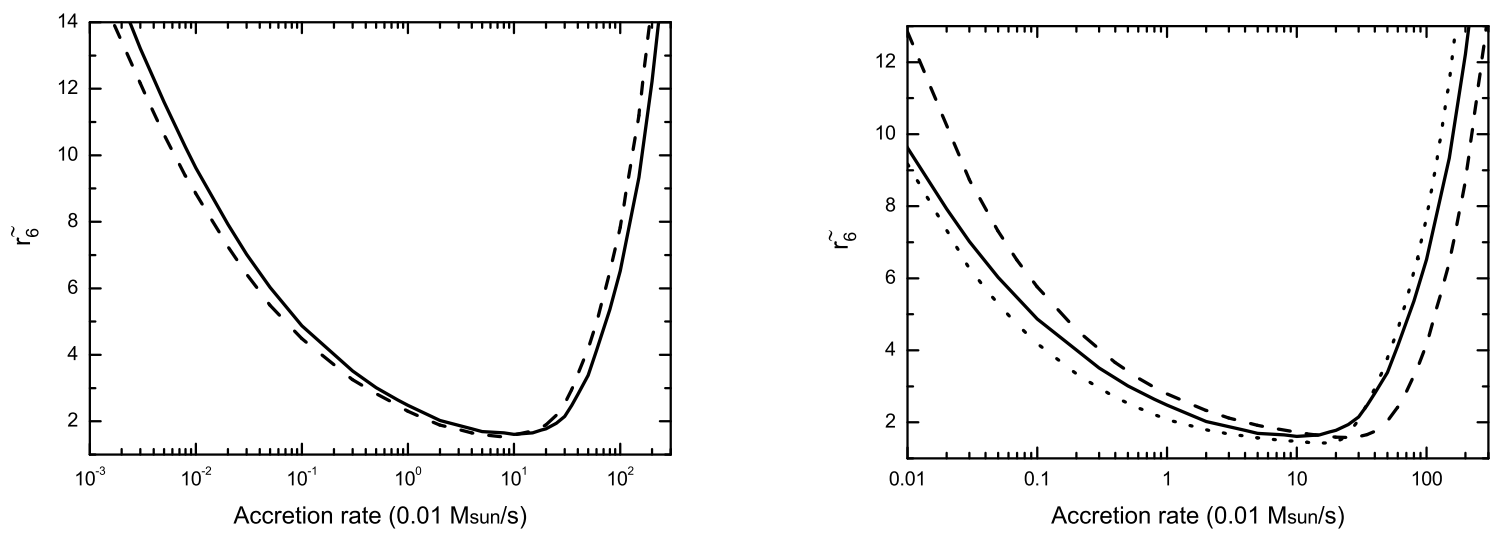

Fig. 6.- (a) Left panel: the radius $\tilde{r}_{6}$ between the inner and the outer disks in the elaborate model with $M=1.4 M_{\odot}$ (solid line), and $M=2.0 M_{\odot}$ (dashed line). (b) Right panel: comparison of $\tilde{r}_{6}$ in different models with $M=1.4 M_{\odot}:$ (1) the elaborate model (solid line), (2) the simple model with $Y_{e}=1$ (dashed line), and (3) the simple model with $Y_{e}=1 / 9$ (dotted line).
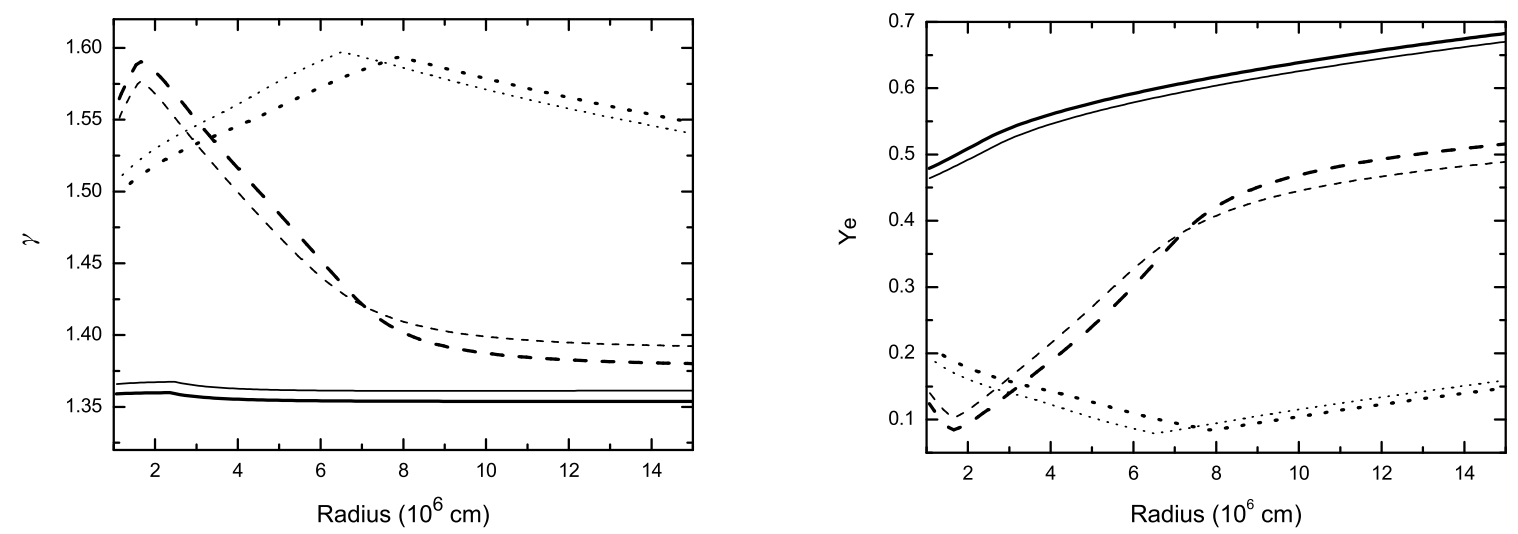

Fig. 7.- (a) Left panel: the "equivalent" adiabatic index $\gamma$ in the elaborate model. (b) Right panel: the electron fraction $Y_{e}$ in the elaborate model as a function of radius. The profiles are shown for three values of the accretion rate and two values of the central neutron star mass: (1) $M=1.4 M_{\odot}$ and $\dot{M}=0.01 M_{\odot} \mathrm{s}^{-1}$ (thin solid line), (2) $M=1.4 M_{\odot}$ and $\dot{M}=0.1 M_{\odot} \mathrm{s}^{-1}$ (thin dashed line), (3) $M=1.4 M_{\odot}$ and $\dot{M}=1.0 M_{\odot} \mathrm{s}^{-1}$ (thin dotted line), (4) $M=2.0 M_{\odot}$ and $\dot{M}=0.01 M_{\odot} \mathrm{s}^{-1}$ (thick solid line), (5) $M=2.0 M_{\odot}$ and $\dot{M}=0.1 M_{\odot} \mathrm{s}^{-1}$ (thick dashed line), and (6) $M=2.0 M_{\odot}$ and $\dot{M}=1.0 M_{\odot} \mathrm{s}^{-1}$ (thick dotted line). 

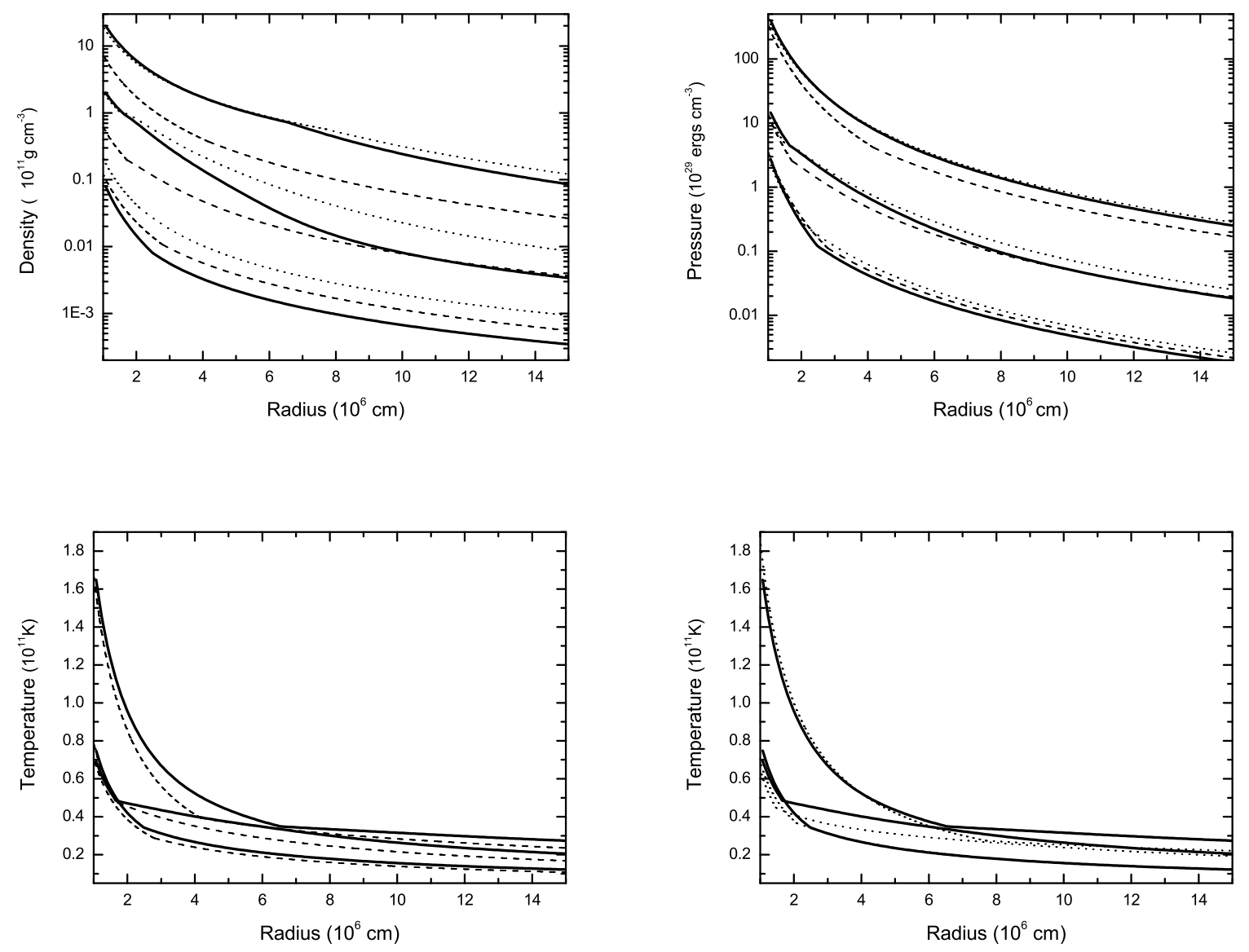

Fig. 8. - The density (in units of $10^{11} \mathrm{~g} \mathrm{~cm}^{-3}$ ), pressure (in units of $10^{11} \mathrm{ergs}^{-3}$ ) and temperature (in units of $10^{11} \mathrm{~K}$ ) of the entire disk in two models for $M=1.4 M_{\odot}$. The profiles include three groups of lines and each group also includes three lines, which are shown for three values of the accretion rate $\dot{M}=0.01 M_{\odot} \mathrm{s}^{-1}, 0.1 M_{\odot} \mathrm{s}^{-1}$ and $1.0 M_{\odot} \mathrm{s}^{-1}$ from bottom to top in these figures. The solution in the simple model with $Y_{e}=1$ is shown by the thin dashed line, and $Y_{e}=1 / 9$ by the thin dotted line. The solution in the elaborate model is shown by the thick solid line. 

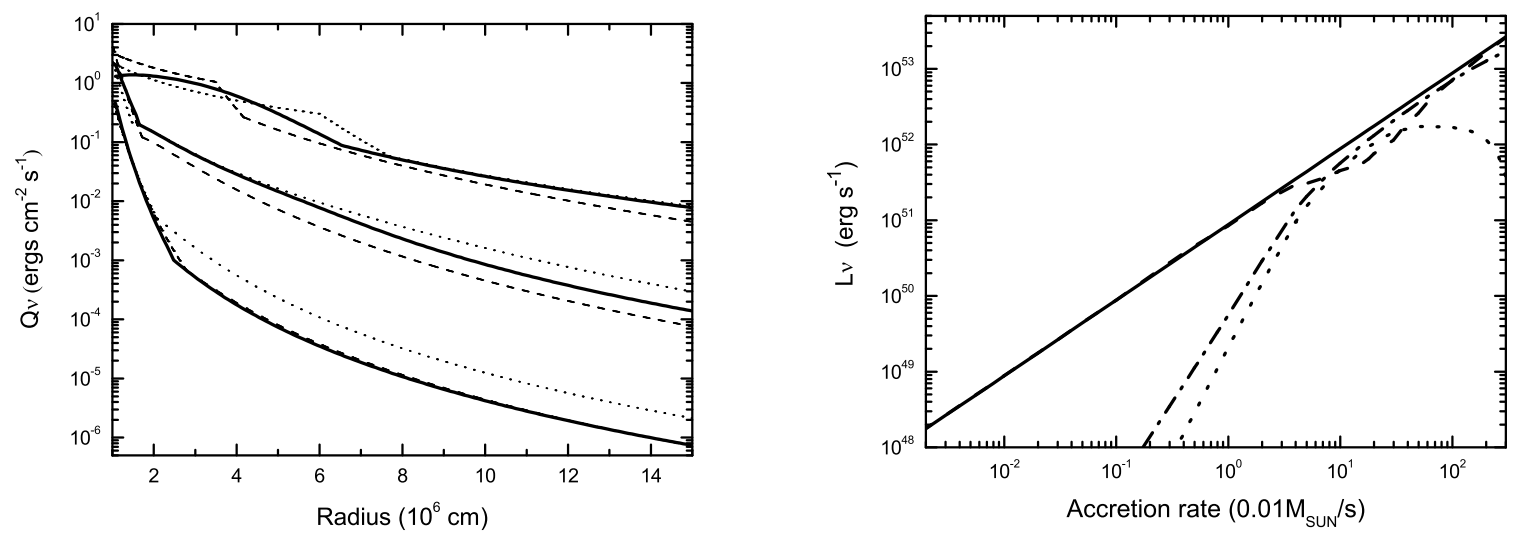

Fig. 9.- (a) Left panel: the neutrino luminosity per unit area (in units of $10^{39} \mathrm{ergs} \mathrm{cm}^{-2} \mathrm{~s}^{-1}$ ) in both the simple model and the elaborate model. The meanings of different lines are the same as those in Fig. 8. (b) Right panel: the neutrino luminosity from the disk in the elaborate model. The meanings of different lines are the same as those in Fig .5. 


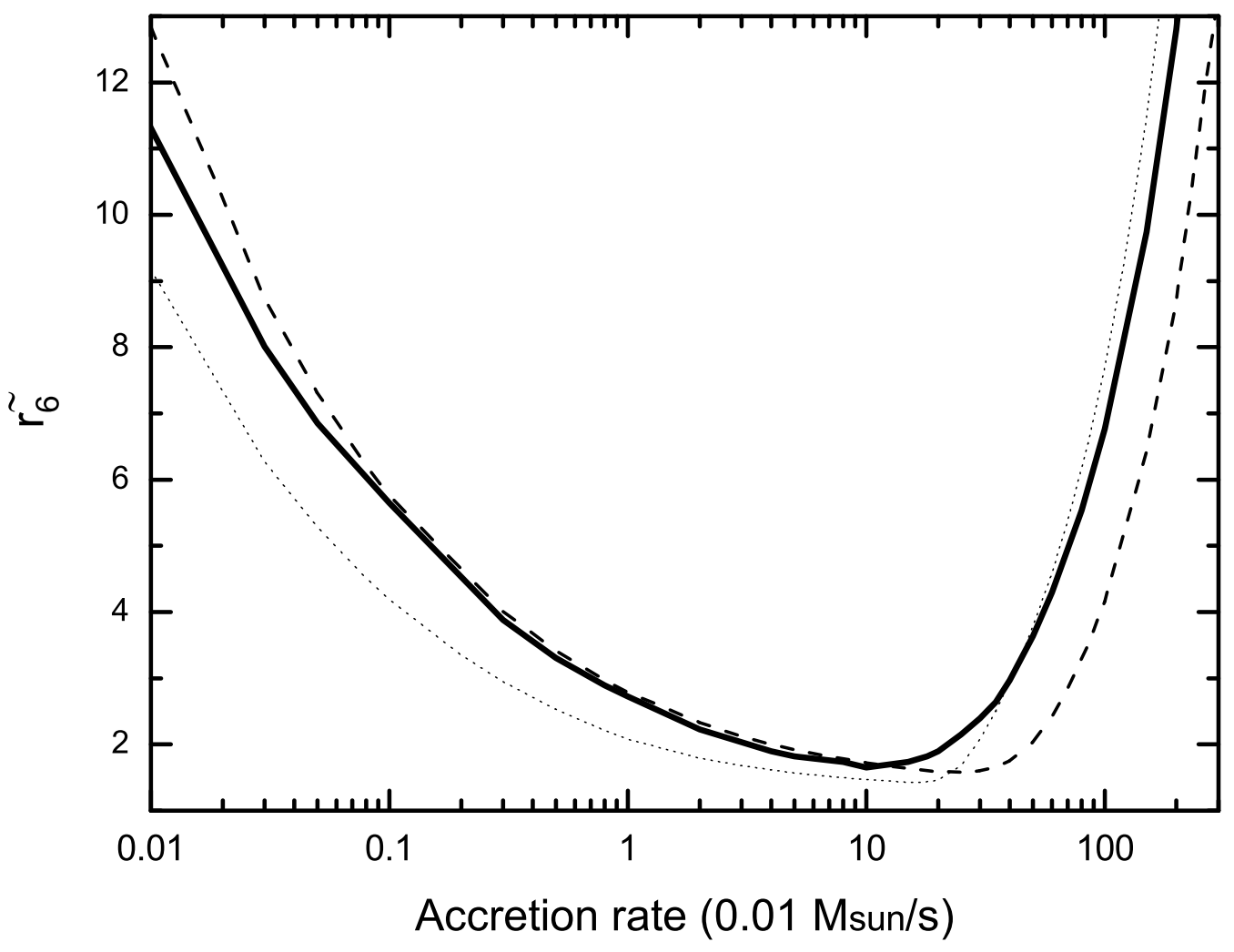

Fig. 10.-Comparison of $\tilde{r}_{6}$ in three models with $M=1.4 M_{\odot}$ : the simple model with $Y_{e}=1$ (dashed line), the simple model with $Y_{e}=1 / 9$ (dotted line), and the third model discussed in $\S 5$ (thick solid line). The solution of $\tilde{r}_{6}$ in the third model is even more consistent with the simple model than that of the elaborate model discussed in $\S 4$. 